\title{
Biofabrication
}

PAPER

CrossMark

OPEN ACCESS

RECEIVED

10 November 2020

REVISED

20 March 2021

ACCEPTED FOR PUBLICATION

9 April 2021

PUBLISHED

26 April 2021

Original content from

this work may be used

under the terms of the

Creative Commons

Attribution 4.0 licence.

Any further distribution

of this work must

maintain attribution to

the author(s) and the title

of the work, journal

citation and DOI.

\section{Bioengineered in vitro 3D model of myotonic dystrophy type 1 human skeletal muscle}

\author{
Xiomara Fernández-Garibay ${ }^{1}\left(10\right.$, María A Ortega $^{1}$, Estefanía Cerro-Herreros ${ }^{2,3,4}$, Jordi Comelles ${ }^{1,5}$, \\ Elena Martínez ${ }^{1,5,6}$, Rubén Artero ${ }^{2,3,4}$, Juan M Fernández-Costa ${ }^{1, *}{ }^{(1)}$ and Javier Ramón-Azcón ${ }^{1,7, *}$ ([) \\ 1 Institute for Bioengineering of Catalonia (IBEC), The Barcelona Institute of Science and Technology (BIST), c/Baldiri Reixac 10-12, \\ E08028 Barcelona, Spain \\ 2 University Institute for Biotechnology and Biomedicine (BIOTECMED), University of Valencia, Dr Moliner 50, E46100 Burjassot, \\ Valencia, Spain \\ 3 Translational Genomics Group, Incliva Health Research Institute, Dr Moliner 50, E46100 Burjassot, Valencia, Spain \\ 4 Joint Unit Incliva- CIPF, Dr Moliner 50, E46100 Burjassot, Valencia, Spain \\ 5 Department of Electronics and Biomedical Engineering, University of Barcelona (UB), c/Martí i Franquès 1-11, E08028 Barcelona, \\ Spain \\ 6 Centro de Investigación Biomédica en Red (CIBER), Av. Monforte de Lemos 3-5, Pabellón 11, Planta 0, E28029 Madrid, Spain \\ 7 Institució Catalana de Reserca I Estudis Avançats (ICREA), Passeig de Lluís Companys, 23, E08010 Barcelona, Spain \\ * Authors to whom any correspondence should be addressed. \\ E-mail: jfernandez@ibecbarcelona.eu and jramon@ibecbarcelona.eu \\ Keywords: myotonic dystrophy, skeletal muscle, tissue engineering, hydrogel micropatterning, 3D cell culture \\ Supplementary material for this article is available online
}

\begin{abstract}
Myotonic dystrophy type 1 (DM1) is the most common hereditary myopathy in the adult population. The disease is characterized by progressive skeletal muscle degeneration that produces severe disability. At present, there is still no effective treatment for DM1 patients, but the breakthroughs in understanding the molecular pathogenic mechanisms in DM1 have allowed the testing of new therapeutic strategies. Animal models and in vitro two-dimensional cell cultures have been essential for these advances. However, serious concerns exist regarding how faithfully these models reproduce the biological complexity of the disease. Biofabrication tools can be applied to engineer human three-dimensional (3D) culture systems that complement current preclinical research models. Here, we describe the development of the first in vitro 3D model of DM1 human skeletal muscle. Transdifferentiated myoblasts from patient-derived fibroblasts were encapsulated in micromolded gelatin methacryloyl-carboxymethyl cellulose methacrylate hydrogels through photomold patterning on functionalized glass coverslips. These hydrogels present a microstructured topography that promotes myoblasts alignment and differentiation resulting in highly aligned myotubes from both healthy and DM1 cells in a long-lasting cell culture. The DM1 3D microtissues recapitulate the molecular alterations detected in patient biopsies. Importantly, fusion index analyses demonstrate that 3D micropatterning significantly improved DM1 cell differentiation into multinucleated myotubes compared to standard cell cultures. Moreover, the characterization of the 3D cultures of DM1 myotubes detects phenotypes as the reduced thickness of myotubes that can be used for drug testing. Finally, we evaluated the therapeutic effect of antagomiR-23b administration on bioengineered DM1 skeletal muscle microtissues. AntagomiR-23b treatment rescues both molecular DM1 hallmarks and structural phenotype, restoring myotube diameter to healthy control sizes. Overall, these new microtissues represent an improvement over conventional cell culture models and can be used as biomimetic platforms to establish preclinical studies for myotonic dystrophy.
\end{abstract}




\section{Introduction}

The skeletal muscle tissue is one of the largest organs of the human body, and it is crucial for locomotion, thermogenesis, and metabolism maintenance [1]. This tissue can be affected by several neuromuscular or metabolic disorders, such as myasthenia gravis [2], McArdle disease [3], type 2 diabetes [4, 5], and muscular dystrophies [6]. Muscular dystrophies are genetically inherited degenerative disorders for most of which there is no cure to date. These disorders share muscle weakness and wasting as common symptoms; however, muscular dystrophies are very heterogeneous, differing in age of onset, rate of progression, inheritance pattern, and type of muscles that are affected [6-8]. The intrinsic heterogenicity of these diseases makes the development of new therapies especially challenging since it is expected that each patient would have a different response to treatments.

Drug development for new muscular dystrophy therapies usually involves the use of in vitro cell culture assays and in vivo animal models in phases before clinical trials. These strategies have long contributed to drug discovery by analyzing specific features of biological processes and identifying the molecular causes of certain diseases [9]. Still, both have significant shortcomings that make it challenging to obtain physiologically relevant results for humans. For instance, conventional in vitro models involve 2D cell monolayers cultured on flat and rigid substrates. These models do not emulate the complexity of real tissues, which have a three-dimensional (3D) structural organization of cells surrounded by each other and an extracellular matrix [10]. On the other hand, functional 3D tissues representing tissue physiology can be found in animal models. The use of animal models in pharmacological research raises several ethical concerns. Moreover, in vivo models often fail to predict the clinical efficacy of drugs. This is due to species-specific differences that limit the extrapolation of animal data to human conditions [11]. It has been estimated that following this drug development pathway, only $11.8 \%$ of drugs entering clinical trials become approved, generating a cost of billions of dollars for newly authorized drugs [12, 13]. To accelerate preclinical research, in vitro studies could be complemented by human 3D culture systems. These in vitro systems consist of patient-derived bioengineered skeletal muscle tissues that offer a better representation of the environment of living tissues with a particular 3D architecture [14].

Skeletal muscle architecture is characterized by bundles of aligned muscle fibers (myofibers). These fibers are formed by the fusion of muscle precursor cells (myoblasts) into multinucleated myotubes, which later become mature myofibers [15]. Because of this complex architecture, engineering skeletal muscle tissues requires a specific organization of muscle precursor cells. These are usually embedded in a suitable biomaterial scaffold, promoting differentiation of myoblasts to form aligned myotubes [16]. Moreover, the biomaterial should provide cells with an appropriate $3 \mathrm{D}$ growth environment, optimal oxygen levels, effective nutrient transport, and mechanical integrity over an extended culture period [10]. Hydrogels of natural origin, such as collagen, gelatin, or fibrin, have been widely used as biomaterial scaffolds for engineered skeletal muscle [17]. This is due to their high water content and the presence of cell adhesion and degradation motifs, which allow cell growth and matrix remodeling. Among these hydrogels, gelatin methacryloyl (GelMA) and other methacrylated biomaterials have been used to encapsulate cells in defined 3D structures due to their photocrosslinkable properties [18]. Also, these hydrogels can further be combined with non-degradable biomaterials, such as alginate or carboxymethyl cellulose. The addition of these polysaccharides to GelMA composite hydrogels enhances their mechanical properties and supports long-term culture of myotubes [19-21].

In this work, we focused on developing the first bioengineered 3D model of myotonic dystrophy type 1 (DM1) human skeletal muscle tissue. DM1 is a life-threatening and chronically debilitating disorder, which is the most common hereditary myopathy in adults (for a recent review of DM1 hallmarks see [22]). The genetic cause of DM1 is a dynamic mutation that expands the cytosine-thyminguanine (CTG) triplet repeat in the $3^{\prime}$ non-translated region of the Dystrophia Myotonica Protein Kinase (DMPK) gene [23]. Cytosine-uracil-guanine (CUG) repeat RNAs accumulate in nuclear foci. This mutant $D M P K$ RNA causes toxic gene misregulation events at the level of gene expression [24, 25], translation [26], gene silencing [27-30], alternative splicing [3133], and polyadenylation of subsets of transcripts [34]. Muscleblind-like (MBNL) proteins, a family of alternative splicing regulators, are sequestered in these CUG foci, and concomitantly their molecular function is impaired [35]. This results in abnormal alternative splicing events directly related to several disease characteristic symptoms, such as muscle weakness and hyper contraction.

Although significant advances have been made in studying the molecular causes of DM1, there is still no effective treatment for patients. Animal models, such as mouse [36], fly [37-39], and zebrafish [40], have been used to evaluate different therapeutic candidates [41-45]. Among these, therapeutic gene modulation is a promising strategy that has the objective of regulating the endogenous expression of a gene to mitigate a certain disease [46]. Following this approximation, it has been demonstrated that microRNAs (miRs) that inhibit MBNL translation can be silenced by antisense oligonucleotides (antagomiRs). Concretely, specific blocking of miR-218 and miR-23b has resulted in increased MBNL protein levels and rescue of 
mis-splicing events in DM1 human myoblasts [46] and $\mathrm{HSA}^{\mathrm{LR}}$ DM1 model mice, where low toxicity, high efficacy, and long-lasting biological effects were observed [46, 47]. While these are encouraging results, the effectiveness of treatments for DM1 still needs to be investigated in clinical phases.

Here, we describe a method to fabricate a bioengineered 3D DM1 skeletal muscle tissue model using transdifferentiated myoblasts from patient-derived fibroblasts. These cells were encapsulated in micropatterned GelMA-carboxymethyl cellulose methacrylate (CMCMA) hydrogels on top of functionalized glass coverslips. Cells were cultured for up to three weeks obtaining aligned myotubes with diseaseassociated molecular and structural phenotypic features. Remarkably, cell encapsulation in micropatterns improved DM1 myotube formation compared to traditional 2D cultures. Moreover, the analysis of $3 \mathrm{D}$ reconstructed myotubes showed that DM1 myotubes have a thinner phenotype than myotubes from healthy control cells. Additionally, as a proofof-concept, we showed that antagomiR treatment for miR-23b could rescue MBNL expression and myotube diameter in 3D DM1 human myotubes. Overall, we demonstrate that patient-derived bioengineered DM1 skeletal muscle microtissues represent valuable in vitro tools for preclinical research. Finally, the developed fabrication method for DM1 could easily be translated for drug development and other studies on muscular dystrophies.

\section{Experimental procedure}

\subsection{Cell culture}

Immortalized human fibroblasts from unaffected control and DM1 patient (carrying 1300 CTG repeats quantified in the blood cells) [48] were kindly provided by Dr Denis Furling and Dr Vincent Mouly (Institute of Myology, Paris). Fibroblast cells were grown in Dulbecco's modified eagle medium (DMEM, $4.5 \mathrm{~g} \mathrm{l}^{-1}$ glucose, Gibco) supplemented with $1 \%$ penicillin-streptomycin $(\mathrm{P} / \mathrm{S}$, $10000 \mathrm{U} \mathrm{ml}^{-1}$, Thermofisher) and 15\% fetal bovine serum (Gibco). Transdifferentiation into myoblastlike cells was induced by turning on the myogenic program, forcing MyoD expression when culturing cells in muscle differentiation medium (MDM). MDM consisted of DMEM with $4.5 \mathrm{~g} \mathrm{l}^{-1}$ glucose, $1 \% \mathrm{P} / \mathrm{S}, 2 \%$ horse serum, $1 \%$ apo-transferrin $\left(10 \mathrm{mg} \mathrm{ml}{ }^{-1}\right), 0.1 \%$ insulin $\left(10 \mathrm{mg} \mathrm{ml}^{-1}\right)$, and $0.02 \%$ doxycycline $\left(10 \mathrm{mg} \mathrm{ml}^{-1}\right)$. In all cases, cells were grown at $37{ }^{\circ} \mathrm{C}$ in a humidified atmosphere containing $5 \% \mathrm{CO}_{2}$. For cell differentiation in $2 \mathrm{D}$, fibroblasts were seeded at 12000 cells $\mathrm{cm}^{-2}$ in 12well plates containing glass coverslips coated with $50 \mu \mathrm{g} \mathrm{ml}^{-1}$ collagen type I and were cultured in growth medium. Once the cells were confluent, the growth medium was replaced by MDM to induce the myogenic program. Fusion index was analyzed in cultures differentiated for seven days.

\subsection{Microstructured stamps fabrication}

Microstructured poly(dimethylsiloxane) (PDMS), Sylgard $^{\mathrm{TM}}$ Elastomer base and curing agent) stamps were fabricated by replica molding of silicon wafer molds.

\subsubsection{Microfabrication of silicon molds}

Silicon wafer molds were fabricated by standard photolithography techniques using SU-8 negative photoresist. Briefly, silicon wafers ( $4^{\prime \prime}$ n-type $<111>$, MicroChemicals $\mathrm{GmbH}$ ) were cleaned in a plasma chamber for $20 \mathrm{~min}$ at $6.8 \mathrm{~W}$. After that, wafers were heated for $5 \mathrm{~min}$ in a hotplate at $95{ }^{\circ} \mathrm{C}$ to dehydrate them. Negative resist SU-8 2100 (2100, MicroChem) was spin-coated in two steps to obtain structures of $100 \mu \mathrm{m}$ in height. To make the patterns, a mask aligner (SÜSS Microtec, MJB4) was set to $240 \mathrm{~mJ} \mathrm{~cm}{ }^{-2}$ energy radiation at $365 \mathrm{~nm}$, and a highquality emulsion flexible film was used as a photomask (JD Photodata, UK). Irradiation time was set to 20 s. Cross-linkage of negative-tone exposed regions of SU-8 was subject to a hotplate cycle of $65{ }^{\circ} \mathrm{C}$ and $95{ }^{\circ} \mathrm{C}$ for 5 and $20 \mathrm{~min}$, respectively. The labile photoresist was removed by developing the crosslinked patterns immersing the wafer in SU-8 developer (MicroChem) for $10 \mathrm{~min}$. Then, the action of 2-propanol stopped development. After a quick $\mathrm{N}_{2}$ blow, we heated the molds for $60 \mathrm{~min}$ at $120^{\circ} \mathrm{C}$. As a final step, the SU-8 patterned silicon molds had to undergo silanization to obtain hydrophobic surfaces and avoid permanent bonding with the PDMS.

\subsubsection{Replica molding of microstructured PDMS} stamps

The polymer elastomer base and curing agent were weighted in a 10:1 ratio to obtain microstructured stamps of $6 \mathrm{~mm}$ in diameter, with channels of $100 \mu \mathrm{m}$ and $200 \mu \mathrm{m}$ of width and height. After mixing thoroughly, we degassed the polymer in a vacuum chamber. PDMS was poured on the patterned silicon substrate and cured at $80^{\circ} \mathrm{C}$ for $4 \mathrm{~h}$. Finally, PDMS was detached and cut off using a $5 \mathrm{~mm}$ biopsy punch. PDMS stamps were cleaned by sonication in Milli-Q water and 2-propanol for $5 \mathrm{~min}$ and dried using $\mathrm{N}_{2}$ flow before using them for tissue fabrication.

\subsection{Prepolymer preparation}

GelMA (figure 1(e)) and CMCMA (figure 1(g)) were synthesized as previously described [19]. These prepolymer precursors and the photoinitiator lithium phenyl (2,4,6-trimethylbenzoyl) phosphinate (LAP, TCI Europe N.V.) were dissolved in MDM at $65^{\circ} \mathrm{C}$ for $2 \mathrm{~h}$. The concentrations of GelMA, CMCMA and LAP were fixed to obtain final concentrations of $5 \%$, $1 \%$ and $0.1 \%(\mathrm{w} / \mathrm{v})$, respectively. 
(a)<smiles>C=C(C)C(=O)OCCC[Si](OC)(OC)OC</smiles>

3-(Trimethoxysilyl)propyl methacrylate (TMSPMA) (b)
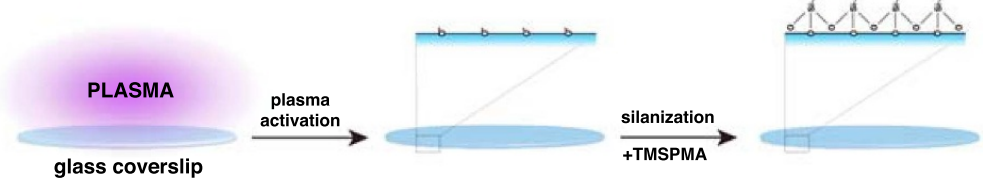

(d)
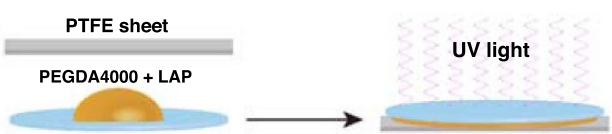

polymerization

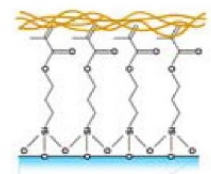

(f)
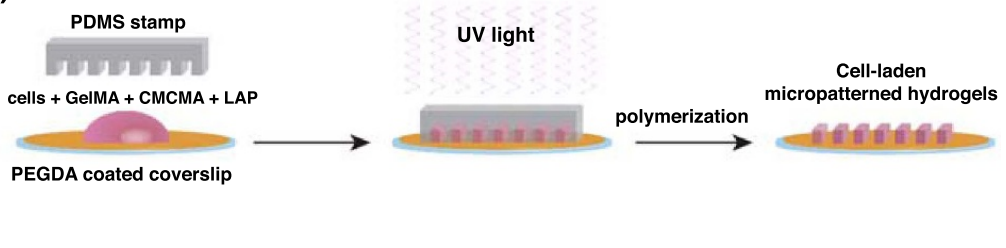

(h)

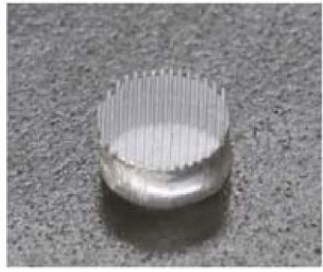

(i)

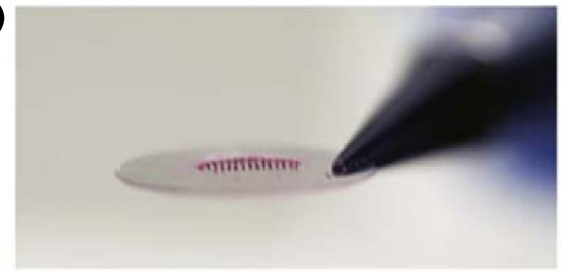

Carboxymethyl cellulose methacrylate (CMCMA)

Figure 1. Fabrication protocol to obtain 3D skeletal muscle microtissues. (a), (c), (e), (g) Chemical structures of (a) 3-(trimethoxysilyl)propyl methacrylate (TMSPMA), (c) poly(ethylene glycol) diacrylate (PEGDA), (e) gelatin methacryloyl (GelMA) and (g) carboxymethyl cellulose methacrylate (CMCMA). (b) Schematic of silanization of glass coverslips by plasma activation and treatment with TMSPMA. (d) Silanized coverslips were coated with a PEGDA layer. PEGDA was polymerized by UV light exposure in the presence of a photoinitiator, lithium phenyl (2,4,6-trimethylbenzoyl) phosphinate (LAP). (f), (h), (i) Photomold patterning of cell-laden GelMA-CMCMA hydrogels. A microstructured poly(dimethylsiloxane) (PDMS) stamp (h) was used to fabricate cell-laden micropatterned hydrogels on top of PEGDA-coated glass coverslips (i) by polymerization with UV light.

\subsection{Cell encapsulation in $3 \mathrm{D}$ microstructured hydrogels}

Cell-laden 3D microstructured hydrogels were fabricated on top of glass coverslips using a photomold patterning technique, as described in [20].

\subsubsection{Glass coverslip functionalization}

Glass coverslips were previously treated with 3-(trimethoxysilyl)propyl methacrylate (TMSPMA, Aldrich) (figure 1(a)) and coated with a layer of polyethylene glycol diacrylate (PEGDA, MW 4000 Da, Sigma-Aldrich) (figure 1(c)) by free radical polymerization. First, clean glass coverslips were activated by oxygen plasma at $29.6 \mathrm{~W}$ for $30 \mathrm{~s}$. Immediately after plasma treatment, the glass surface was covered with a freshly prepared silanization solution (TMSPMA diluted in ethanol at 1:50 and mixed with $3 \%$ acetic acid) for $1 \mathrm{~h}$. Then, coverslips were thoroughly washed with ethanol and dried (figure 1(b)).
For PEGDA coating, we prepared a solution of 20\% (w/v) PEGDA and 2\% (w/v) LAP in Milli-Q water by dissolving in a water bath at $60{ }^{\circ} \mathrm{C}$ for $30 \mathrm{~min}$. For each coverslip, a $5 \mu \mathrm{l}$ drop was placed on a Teflon surface. Then, the silanized side of the coverslip was carefully pressed on top. Finally, samples were exposed to UV for 2 min using a UVP Crosslinker (model CL-1000 1, 365 nm, 40 W, Analytik Jena US), washed several times with Milli-Q water, and thoroughly dried (figure $1(\mathrm{~d})$ ).

\subsubsection{Cell encapsulation}

To encapsulate cells in micropatterned hydrogels, we mixed the prepolymer solution with a cell suspension of either control or DM1 cells in MDM to obtain a final concentration of $2.5 \times 10^{7}$ cells $\mathrm{ml}^{-1}$. Then, an $8 \mu \mathrm{l}$ drop of the cell-laden prepolymer was placed on a PEGDA-coated glass coverslip, and a PDMS stamp (figure $1(\mathrm{~h})$ ) was pressed lightly on top, filling the microchannels with the solution. The hydrogels were 
crosslinked by UV exposure of $30 \mathrm{~s}$ using the UVP Crosslinker. MDM was added to each sample, and stamps were carefully removed after $20 \mathrm{~min}$ of incubation at $37{ }^{\circ} \mathrm{C}$ (figures $1(\mathrm{f})$ and (i)). Encapsulated cells were differentiated for up to 21 days, with culture media being replaced every two days.

\subsection{Hydrogel characterization}

\subsubsection{Stiffness measurements}

The bulk stiffness of GelMA-CMCMA hydrogels was analyzed by uniaxial compression tests using a Zwick Z0.5 TN instrument (Zwick-Roell, Germany) with a $5 \mathrm{~N}$ load cell. Hydrogels were fabricated as described in [20]. For each sample, $300 \mu \mathrm{l}$ of the prepolymer solution were placed in a 48-well plate and exposed to UV light for $30 \mathrm{~s}$. Cylindrical samples were cut using a $10 \mathrm{~mm}$ diameter biopsy punch. Samples were tested at room temperature (RT) up to $30 \%$ final strain (deformation), using the following parameters: $0.1 \mathrm{mN}$ preload force and $20 \% \mathrm{~min}^{-1}$ strain rate. Force-deformation graphs were obtained using the TestXpert (Zwick-Roell) software. Values for the compressive modulus were automatically calculated from the slope of the linear region corresponding to $10 \%$ $20 \%$ deformation (strain) with the TestXpert software.

To obtain Young's modulus of the micropatterned GelMA-CMCMA hydrogels in liquid conditions, samples were photomolded as described before (see section 2.4). After $24 \mathrm{~h}$ of incubation at $37^{\circ} \mathrm{C}$, the stiffness was measured by atomic force microscopy (AFM) following a previously described protocol [49, 50]. Briefly, indentation measurements were conducted using a NanoWizard ${ }^{\circledR} 4$ Bioscience AFM (JPK Instruments) mounted onto a Nikon $\mathrm{Ti}$ inverted microscope. Silicon nitride pyramidal tips (NanoWorld) with nominal spring constants of $0.08 \mathrm{Nm}^{-1}$ were used. Series of ten indentations at a frequency of $0.05 \mathrm{~Hz}$ (10 $\mu \mathrm{m}$ amplitude) were performed in different positions of the GelMA-CMCMA micropatterns. Finally, the Hertz model for a pyramidal tip was fitted to the measured force-distance curves, using the JPK data analysis software.

\subsubsection{Scanning electron microscopy (SEM) imaging}

Hydrogels for SEM imaging were fabricated following the same protocol as for compression measurements. After $24 \mathrm{~h}$ of incubation, the hydrogels were washed with Milli-Q water and fixed for $1 \mathrm{~h}$ in a $2.5 \%$ glutaraldehyde solution (Sigma-Aldrich). Following several washes, samples were dehydrated by sequential immersion in graded ethanol solutions in Milli-Q water. Washings of 10 min were performed with $50 \%, 70 \%(\times 2), 90 \%(\times 3), 96 \%(\times 3)$ and 99.5\% $(\times 3)$ ethanol. To dry the hydrogels without causing their collapse, samples were placed in a critical point drying chamber (K850, Quorum Technologies, UK), where ethanol was completely replaced by liquid $\mathrm{CO}_{2}$ and gradually heated until $\mathrm{CO}_{2}$ achieved gas phase equilibrium and was slowly drained. After critical point drying, microstructured hydrogels were covered with Au and imaged by ultrahigh-resolution SEM (Nova NanoSEM 230, FEI Company, The Netherlands) operating in a low vacuum mode ( $0.5 \mathrm{mbar}$ of water vapor pressure).

\subsection{Cell viability assay}

Cell viability was evaluated $24 \mathrm{~h}$ after encapsulation using the Live/Dead viability/cytotoxicity kit (Invitrogen). Briefly, samples were washed several times with sterile $1 \times$ phosphate buffered saline (PBS) and incubated for $30 \mathrm{~min}$ with a staining solution containing Calcein AM, Ethidium homodimer-1, and Hoescht (Invitrogen) in PBS. Cell viability was calculated as the percentage of living cells with respect to the total cell number analyzed in the $3 \mathrm{D}$ reconstruction of confocal Z-stacks using Imaris software.

\subsection{Antisense oligonucleotide treatment}

AntagomiR oligonucleotides (Creative Biogene) were administrated $72 \mathrm{~h}$ after DM1 microtissue fabrication. MDM was replaced and supplemented with $100 \mathrm{nM}$ of AntagomiRs for miR-sc (control) or miR-23b-3p. The treatment lasted for seven days without replacing the culture media. The antagomiR sequences were: $5^{\prime}-\mathrm{mG}^{*} \mathrm{mG}^{*} \mathrm{mUm}$ AmAmUmCmCmCmUmGmGmCmAmAmUmGm $\mathrm{U}^{*} \mathrm{mG}^{*} \mathrm{~mA}^{*} \mathrm{mU}^{*}-3^{\prime}$-chol (antagomiR-23b-3p), and $5^{\prime}-\mathrm{mC}^{*} \mathrm{~mA} \mathrm{~A}^{*} \mathrm{mGmUmAmCmUmUmUmUmGmUm}$ GmUmA* $\mathrm{mC}^{*} \mathrm{~mA}^{*} \mathrm{~mA}^{*}-3^{\prime}$-chol (sc control). Where $\mathrm{m}$ denotes $2^{\prime}$-O-methyl-modified phosphoramidities, * denotes phosphorothioate linkages, and chol denotes cholesterol groups.

\subsection{Fluorescence in situ hybridization (FISH)}

Bioengineered 3D skeletal muscle tissues were fixed in $4 \%$ paraformaldehyde for $30 \mathrm{~min}$ at RT and washed with $1 \times$ PBS for $10 \mathrm{~min}$. Samples were incubated in the prehybridization buffer $(2 \times$ saline sodium citrate [SSC], 30\% deionized formamide) for $30 \mathrm{~min}$ at RT and hybridized with a Cy3$(\mathrm{CAG})_{7}$-Cy3-labeled probe, diluted 1:200 in hybridization buffer ( $40 \%$ deionized formamide, $2 \times$ SSC, $10 \%$ dextran sulfate, $0.2 \%$ BSA, 2 mM Ribonucleoside Vanadyl Complex (Sigma-Aldrich), $1 \mathrm{mg} \mathrm{ml}^{-1}$ Escherichia coli tRNA, 1\% herring sperm DNA) overnight at $37{ }^{\circ} \mathrm{C}$ in the dark. After hybridization, samples were washed twice with a prehybridization buffer for $30 \mathrm{~min}$ at $42{ }^{\circ} \mathrm{C}$, washed with $1 \mathrm{X}$ PBS, and incubated with DAPI for $15 \mathrm{~min}$. All the incubations were performed in a humidity chamber.

\subsection{Immunofluorescence staining}

Bioengineered muscle tissues were fixed with $10 \%$ formalin solution (Sigma-Aldrich) for $30 \mathrm{~min}$ at 
RT, followed by several washes in PBS. Samples were then permeabilized with PBS-T $(0.1 \%$ Triton$\mathrm{X}$ (Sigma-Aldrich) in PBS), blocked (0.3\% Triton-X, $3 \%$ donkey serum (Sigma-Aldrich) in PBS) for $2 \mathrm{~h}$ at RT, and incubated with primary antibody (supplementary table 1 (available online at stacks.iop.org/ $\mathrm{BF} / 13 / 035035 /$ mmedia)) at $4{ }^{\circ} \mathrm{C}$ overnight. After several PBS-T washes, the samples were incubated for $2 \mathrm{~h}$ with the fluorophore-conjugated secondary antibody (supplementary table 1) at RT. Finally, the samples were counterstained with DAPI $\left(4^{\prime}, 6\right.$-diamidino2-phenylindole, Life Technologies) to detect the nuclei.

For MBNL1 detection, after FISH protocol, samples were incubated with the monoclonal antiMBNL1 primary antibody at $4{ }^{\circ} \mathrm{C}$. The fluorescence signal was amplified using a biotin-conjugated secondary antibody (supplementary table 1) and the VECTASTAIN $^{\circledR}$ Elite $^{\circledR}$ ABC kit (Vector) for $1 \mathrm{~h}$ at RT, followed by PBS-T washes and incubation with either Dylight ${ }^{\circledR} 488$-FITC (1:200, Vector) for $2 \mathrm{~h}$ at RT. Finally, the samples were counterstained with DAPI to detect the nuclei.

\subsection{Imaging}

Light microscopy and live-cell imaging of myotubes were performed using a Zeiss Axio Observer.Z1/7 outfitted with the XL S1 cell incubator. Imaging was performed at $37{ }^{\circ} \mathrm{C}$ and $5 \% \mathrm{CO}_{2}$. Fluorescence images were taken as Z-stacks with a ZEISS LSM800 confocal laser scanning microscope and analyzed using the Imaris microscope image analysis software (Oxford instruments) and the Fiji image processing package, a distribution of Image J [51, 52].

\subsection{Fusion index and myotube size analysis}

Z-stacks were analyzed as 3D images using the Imaris software. Fusion index was determined as the percentage of nuclei in myotubes $(\geqslant 2$ myonuclei) with respect to the total number of nuclei, in myotubes expressing sarcomeric $\alpha$-actinin (SAA). The diameter of individual 3D myotubes was obtained by the object-oriented bounding box statistical variable (BoundingBoxOO Length A), which measures the length of the object's shortest principal axis.

\subsection{RNA extraction, RT-PCR and real-time PCR}

Total RNA from human bioengineered muscles was isolated using Tri-reagent (Sigma) according to the manufacturer's instructions. One microgram of RNA was digested with DNase I (Invitrogen) and reversetranscribed with SuperScript ${ }^{\mathrm{TM}}$ II (Invitrogen) using random hexanucleotides. qRT-PCR was carried out on one nanogram of cDNA template with the FIREPol ${ }^{\circledR}$ EvaGreen ${ }^{\circledR}$ qPCR Mix Plus (Solis Biodyne) and specific primers (supplementary table 2). GAPDH and $A C T B$ were used as endogenous controls. miRNA expression was quantified using specific miRCURY LNA microRNA PCR primers (Qiagen) according to the manufacturer's instructions. Relative gene expression was normalized to miR-103 expression [53]. Expression levels were measured using the QuantStudio 5 Applied Biosystems Real-Time PCR System. Expression relative to the endogenous genes and control group was calculated using the $2^{-\Delta \Delta \mathrm{Ct}}$ method. Pairs of samples were compared using two-tailed Student $t$-tests $(\alpha=0.05)$, applying Welch's correction when necessary. For splicing analyses, 20 ng of cDNA were used in a standard PCR reaction with GoTaq polymerase (Promega). Specific primers were used to analyze alternative splicing of Bridging integrator 1 (BIN1), Nuclear Factor I X (NFIX), and Spectrin Alpha Non-Erythrocytic 1 (SPTAN1) (supplementary table 2).

\subsection{Western blotting}

For total protein extraction, samples were homogenized in RIPA buffer $(150 \mathrm{mM} \mathrm{NaCl}, 1.0 \%$ IGEPAL, $0.5 \%$ sodium deoxycholate, $0.1 \%$ SDS, $50 \mathrm{mM}$ Tris$\mathrm{HCl} \mathrm{pH} \mathrm{8.0)} \mathrm{supplemented} \mathrm{with} \mathrm{protease} \mathrm{and} \mathrm{phos-}$ phatase inhibitor cocktails (Roche Applied Science). Total proteins were quantified with a BCA protein assay kit (Pierce) using bovine serum albumin as the standard concentration range. For the immunodetection assay, $20 \mu \mathrm{g}$ of samples were denatured for $5 \mathrm{~min}$ at $100{ }^{\circ} \mathrm{C}$, electrophoresed on $12 \%$ SDS-PAGE gels, transferred onto nitrocellulose membranes $0.45 \mu \mathrm{m}$ (GE Healthcare), and blocked with 5\% non-fat dried milk in PBS-T $(1 \times$ PBS; $0.05 \%$ Tween 20 , $\mathrm{pH}$ 7.4). Membranes were incubated overnight at $4{ }^{\circ} \mathrm{C}$ with primary mouse anti-MBNL1 (1:200, clone MB1a (4A8), Developmental Studies Hybridoma Bank) or mouse anti-MBNL2 (1:100, clone MB2a (3B4), Developmental Studies Hybridoma Bank). Anti-GAPDH-binding protein-horseradish peroxidase (HRP) antibody (1 h, 1:3500, clone G-9, Santa Cruz) as a loading control. All primary antibodies were detected using HRP-conjugated anti-mouseIgG secondary antibody ( 1 h, 1:5000, Sigma-Aldrich). Immunoreactive bands were detected using $\mathrm{ECL}^{\mathrm{TM}}$ Western blotting substrate (Pierce), and images were acquired with an AMERSHAM ImageQuant 800 (GE Healthcare). Quantification was performed using Image J software. The statistical differences were estimated by the Student's $t$-test $(p<0.05)$ on normalized data.

\subsection{Statistical analysis}

All group data are expressed as mean \pm SEM. The comparisons between groups were performed using Prism 8 software (GraphPad) by two-tailed Student $t$-test $(\alpha=0.05)$, applying Welch's correction when necessary. Differences between groups were considered significant when $P<0.05\left({ }^{*} P<0.05\right.$; ${ }^{* *} P<0.01$; $^{* *} P<0.001$; $\left.^{* * *} P<0.0001\right)$. 


\section{Results and discussion}

\subsection{A fabrication protocol to obtain 3D skeletal muscle microtissues}

The skeletal muscle is a highly organized tissue made up of arrays of aligned, multinucleated myofibers. This complex architecture should be mimicked in vitro to obtain $3 \mathrm{D}$ models that accurately represent the basic features of skeletal muscle tissue. Multinucleated myofibers are formed by fusion and differentiation of muscle precursor cells; therefore, fabrication strategies for tissue engineering should provide effective topographical cues that favor myoblast alignment and fusion. Among these biofabrication techniques to engineer topographical cues, the most used are micropatterning, micromolding, electrospinning, and 3D bioprinting [54]. Previous studies have shown that cells with a tendency to align in vivo (e.g. fibroblasts, murine myoblasts, and endothelial cells) can elongate and align in vitro if they are geometrically confined within a suitable biomaterial [55]. Accordingly, our fabrication protocol was designed to encapsulate human muscle precursor cells in micropatterned hydrogels, which confine cells in narrow hydrogel filaments and induce the formation of elongated myotubes.

As previously described, we used photomold patterning to transfer the features of microstructured PDMS stamps into photocrosslinkable GelMACMCMA hydrogels (figures 1 and 2(a)). We fabricated stamps with channels of $200 \mu \mathrm{m}$ (data not shown) or $100 \mu \mathrm{m}$ of width, height, and spacing. We observed better cell alignment when cells were confined in smaller structures. This was in agreement with other studies where cell alignment and myotube formation were enhanced in the thinnest structures [56]. Besides alignment being favored in narrower patterns, there are other limitations to the dimensions of hydrogels for cell encapsulation. As some studies point out, there is a limited diffusion of oxygen and nutrients in engineered tissues at around $200 \mu \mathrm{m}$ of depth due to the lack of vascularization $[19,57,58]$. Therefore, we fabricated smaller structures to overcome this limitation. Consequently, we decided to use the microstructured stamps with smaller features to fabricate hydrogel filaments of $100 \mu \mathrm{m}$ in width and $5 \mathrm{~mm}$ in length on top of glass coverslips.

Using glass as our substrate, we noticed that some cells adhered to the flat surface between hydrogel filaments outside of the 3D structure (figure 2(b)). This was an unwanted effect since cells in 2D would grow and differentiate at a different speed, creating two separate cell populations. To ensure that cells remained confined within the 3D GelMA-CMCMA hydrogel, the glass surface was previously silanized and coated with a thin layer of PEGDA hydrogel. PEGDA is hydrophilic and inert to protein adsorption [59]; hence, this surface modification highly reduced undesired $2 \mathrm{D}$ cell attachment outside the
GelMA-CMCMA microfilaments fabricated on top of the PEGDA layer (figure 2(c)). Furthermore, GelMACMCMA was covalently attached to PEGDA, which gave more stability to the micropatterned structures [60].

Cell encapsulation in photocrosslinkable hydrogels is achieved by free radical polymerization in the presence of a photoinitiator and UV light. Therefore, after this procedure, cell survival strongly depends on critical factors, including UV wavelength, energy dose, and photoinitiator concentration. When cells are exposed to a low dose of long-wave UV light, the procedure is considered biocompatible [61]. As has been reported, LAP photoinitiator has a high extinction coefficient at $365 \mathrm{~nm}$, which makes it more efficient than other commonly used compounds, such as Irgacure 2659 [19, 62]. This efficiency allowed crosslinking composite hydrogels with a very low photoinitiator concentration and short exposure times (low energy dose). To confirm this, we measured the energy dose received by cells during $30 \mathrm{~s}$ of UV exposure using a wireless power-meter inside the UVP crosslinking chamber, obtaining $0.48 \mathrm{~J} \mathrm{~cm}^{-2}$ $\left(16 \mathrm{~mW} \mathrm{~cm} \mathrm{~cm}^{-2}\right.$ during $30 \mathrm{~s}$ ). This energy dose is very low, considering cell-laden GelMA hydrogels have been crosslinked with energy doses ranging from $0.3 \mathrm{~J} \mathrm{~cm}^{-2}$ to $36 \mathrm{~J} \mathrm{~cm}^{-2}[63,64]$. Moreover, cell cytotoxicity assays (figures $3(\mathrm{a})-(\mathrm{h})$ ) showed that more than $88 \%$ of encapsulated cells remained viable $24 \mathrm{~h}$ after UV exposure (figure 3(i)).

Although both 3D bioprinting and photomold patterning are valuable biofabrication strategies for generating cell-laden hydrogel filaments, photomold patterning offered several advantages compared to our previous 3D bioprinted model [19], such as reproducibility, amount of material required, or the final cost of the procedure.

Human skeletal muscle tissue models have been developed from primary and immortalized human myoblasts and human induced pluripotent stem cells [65]. Bioengineered 3D in vitro models require large numbers of cells, especially for high-throughput drug screening platforms. This represents a challenge for developing disease models, where it is necessary to incorporate patient cells coming from muscle biopsies. A major problem of using primary myoblasts from adult DM1 patients is their limited proliferative capacity due to premature replicative senescence [66]. Besides, muscle biopsies are invasive and not always available. Arandel et al overcame these limitations by developing transdifferentiated myotubes cell lines [48]. These cell lines consisted of immortalized skin fibroblasts that were converted into multinucleated myoblasts by forcing the overexpression of the myogenic regulator factor MYOD1. Here, we fabricated 3D skeletal muscle microtissues from immortalized fibroblasts obtained from a healthy control individual and a DM1 patient. Cell-laden hydrogels were cultured in MDM containing doxycycline and a 

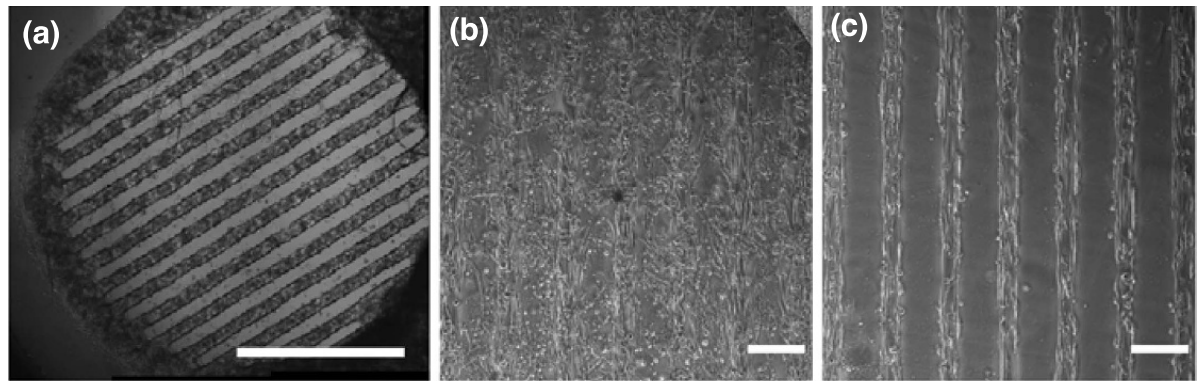

Figure 2. Effect of PEGDA coating on micromolded hydrogels. (a) Phase contrast image of cell-laden micropatterned hydrogels (scale bar: $2.5 \mathrm{~mm}$ ). (b), (c) Representative phase contrast images of cell-laden hydrogels without (b) and with (c) PEGDA coating (scale bars: $200 \mu \mathrm{m}$ ).
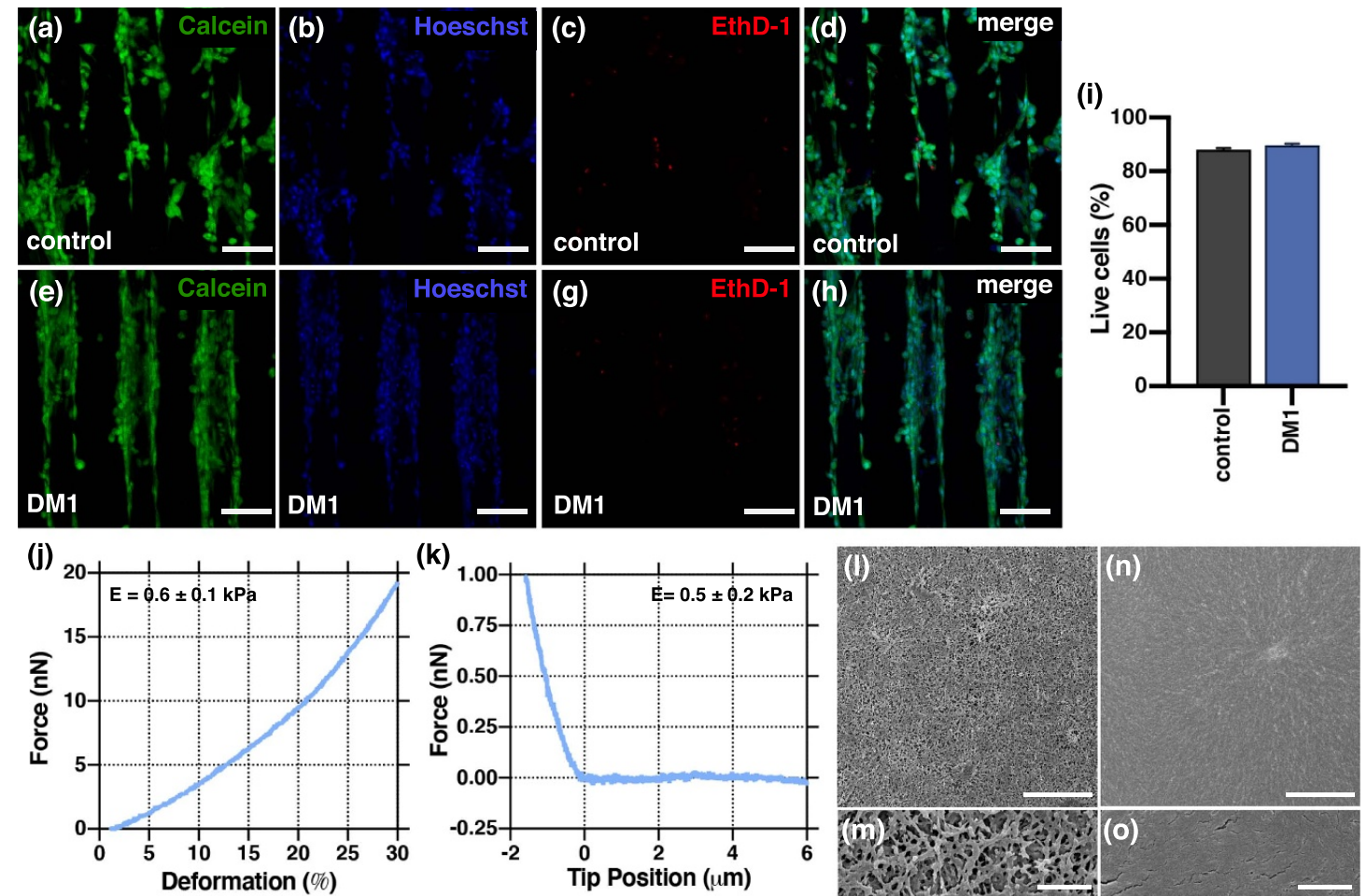

Figure 3. (a)-(h) Representative images of live/dead staining from healthy control (a)-(d) and DM1 cells (e)-(h) $24 \mathrm{~h}$ after cell encapsulation. Live cells were stained with Calcein AM (green), nuclei were stained by Hoescht (blue), and dead cells (red) were stained with Ethidium homodimer-1 (EthD-1) (Scale bars: $100 \mu \mathrm{m}$ ). (i) Graph showing the cell viability in micromolded hydrogels represented as the live cells over the total cell number (mean \pm SEM). (j) Example of a typical force-deformation curve obtained by uniaxial compression tests on bulk GelMA-CMCMA hydrogels. Young's modulus $(\mathrm{E})=0.6 \pm 0.1 \mathrm{kPa}(\mathrm{k})$ typical force-displacement curves of the AFM tip indenting the surface of the GelMA-CMCMA microstructured hydrogels.

$\mathrm{E}=0.5 \pm 0.2 \mathrm{kPa}$ (Mean values $\pm \mathrm{SEM})$. (1)-(o) Scanning electron microscopy (SEM) images of GelMA-CMCMA (l), (m) and PEGDA (n), (o) hydrogels. Scale bars: (1), (n) $5 \mu \mathrm{m},(\mathrm{m})$, (o) $1 \mu \mathrm{m}$.

low serum concentration from the moment of encapsulation. With this culture media, doxycycline activated the myogenic program and transdifferentiation into myoblast-like cells. Additionally, the reduced serum concentration slowed down cell proliferation, inducing a shift to a differentiation state. Furthermore, myoblasts need to be close to recognize each other and fuse into myotubes. Since cell proliferation was limited by the MDM, working with a high cell density $\left(2.5 \times 10^{7}\right.$ cells $\left.\mathrm{ml}^{-1}\right)$ was key in obtaining multinucleated myotubes. In summary, we have developed a reproducible method to generate $3 \mathrm{D}$ skeletal muscle microtissues from human cells. In this protocol, we applied a photomold patterning technique to encapsulate cells in GelMA-CMCMA hydrogel scaffolds.

\subsection{Physical properties of GelMA-CMCMA hydrogels}

Skeletal muscle tissue engineering requires culture time for myoblast fusion and myotube maturation. Additionally, engineered tissues should remain viable for several weeks to be valuable for drug screening applications. For these reasons, the biomaterials used as scaffold need to resist enzymatic degradation by encapsulated cells and other stress 
cues. We previously showed that degradation of GelMA hydrogels could be slowed down by fabricating composites that include a small percentage of a non-degradable polymer [19]. GelMA contains cell adhesion and degradation sites that allow cells to attach and remodel the matrix, a necessary feature for cell spreading and fusion. CMCMA is a biocompatible cellulose derivate that is nondegradable by mammalian cells [67]. Therefore, its incorporation into the hydrogel formulation provided more structural stability over time. In fact, in our previous works, we have demonstrated that this composite hydrogel allowed long-lasting culture and differentiation of murine skeletal muscle cells $[20,68]$.

The bulk stiffness of GelMA-CMCMA hydrogels was measured using uniaxial compression tests, obtaining a Young's modulus of $0.6 \pm 0.1 \mathrm{kPa}$ (figure $3(\mathrm{j})$ ). To calculate the stiffness of our micropatterned hydrogels in liquid conditions, we performed AFM microindentation measurements (figure $3(\mathrm{k})$ ). The obtained values were comparable $(\mathrm{E}=0.5 \pm 0.2 \mathrm{kPa})$, corresponding to soft hydrogels in the range of $0.5-0.7 \mathrm{kPa}$. Some studies suggest that the optimal stiffness for myotube maturation is higher, being closer to the native skeletal muscle tissue $(\sim 12 \mathrm{kPa})[69,70]$. However, these experiments were performed by seeding cells on top of $2 \mathrm{D}$ substrates. In contrast, the stiffness of hydrogels for 3D skeletal muscle models varies depending on the biomaterials used but lean towards softer hydrogels. For instance, the encapsulation of human myoblasts in fibrin-based hydrogels is performed in biomaterials with Young's modulus of $1 \pm 0.1 \mathrm{kPa}$ [71]. Similarly, 3D studies performed with GelMA and $\mathrm{C} 2 \mathrm{C} 12$ murine cells indicated that softer hydrogels in the range of $1-3 \mathrm{kPa}$ lead to more elongated cell morphology, better cell spreading, and greater differentiation in $3 \mathrm{D}$ structures $[20,56]$. In the case of micropatterned GelMA-based hydrogels, stiffer hydrogels can be obtained by modifying fabrication conditions to increase the crosslinking degree. We previously studied cell morphology and spreading in micropatterned GelMA-CMCMA hydrogels with different stiffness values [20]. Stellate morphology indicates that cells can move better within the material, which is essential for cell fusion. We observed that cells had a stellate morphology in softer hydrogels, while most cells were round when encapsulated using longer UV exposure times. Furthermore, longer UV exposure times or higher photoinitiator concentration may affect cell viability (see section 3.1). Moreover, highly crosslinked hydrogels result in densely packed fibrillar structures that reduce water uptake and hinder nutrient diffusion and transport $[19,72,73]$. The fibrillar nanostructure of our photomolded GelMA-CMCMA hydrogels was observed using SEM (figures 3(l) and $(\mathrm{m}))$. The images showed an interconnected porous network that allows the diffusion of growth factors and other biological substances through the hydrogel. Conversely, PEGDA hydrogel analysis in SEM microscopy revealed that it is formed by a dense nonporous matrix (figures $3(\mathrm{n})$ and $(\mathrm{o})$ ). This characteristic structure in the PEGDA layer helps to avoid cell growth and improves the adhesion and attachment of the GelMA-CMCMA scaffold. Altogether, the physical properties of GelMA-CMCMA hydrogel scaffolds are appropriate for supporting long-term cell culture of skeletal muscle tissue.

\subsection{D model of DM1 retains molecular features of the disease and provides a new structural phenotype for preclinical research}

In this work, we fabricated 3D skeletal muscle tissue from human immortalized transdifferentiated myoblast-like cells that present molecular and phenotypical features of DM1. By evaluating 2D cell cultures, Arandel et al confirmed that patient-derived cells present nuclear RNA aggregates (foci) of expanded CUG repeats, a hallmark feature of DM1. They also showed that DM1 cells had a reduced fusion capability and formed smaller myotubes compared to healthy controls in 2D [48]. To validate the use of this cell line for our bioengineered DM1 model, we analyzed if nuclear RNA aggregates were also observed in 3D. By performing FISH and immunofluorescence analyses, we demonstrated the presence of CUGexp-RNA foci and their colocalization with MBNL1 (figure 4), confirming that these cells retain DM1-associated molecular characteristics and are suitable for generating in vitro 3D models for the disease.

To evaluate fusion capability in $3 \mathrm{D}$, we assessed confocal images of 2D cultures and bioengineered microtissues from both control and DM1 myotubes. After seven days in differentiation conditions, we performed immunofluorescence stainings for myosin heavy chain 7, SAA, and nuclei (figures 5(a)-(p)). Then, we used confocal images to calculate the fusion index, which is an indicator of differentiation quality in skeletal muscle cell cultures [74]. As expected, DM1 myoblasts in 2D culture had a significantly lower fusion index than healthy controls. Unexpectedly, when cells were encapsulated in 3D GelMACMCMA hydrogels, there were no differences in differentiation between healthy controls and DM1 cells, obtaining high fusion index values for both cases (figure 5(q)). Remarkably, 3D micropatterning resulted in the formation of long, multinucleated myotubes from DM1 patient-derived cells after seven days of culture, which is not possible to generate in standard cell cultures.

Although the fusion index of 3D DM1 myotubes was comparable to healthy myotubes, we observed structural differences, which prompted us to analyze the myotube diameter. Bioengineered skeletal muscle microtissues were differentiated for 7,14 , or 21 days before immunostaining for confocal imaging. 

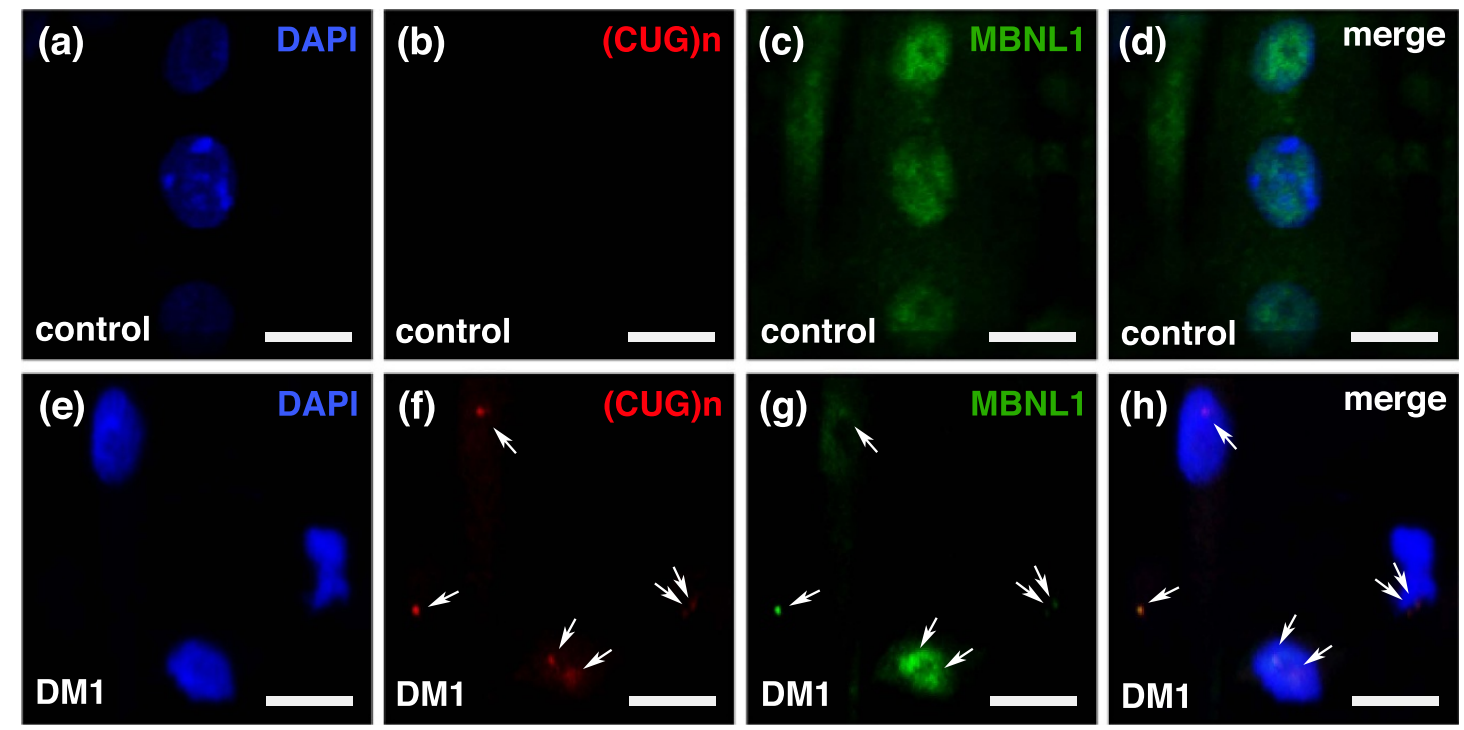

Figure 4. Encapsulated DM1 cells present disease-associated molecular phenotypes. Representative confocal images of (a)-(d) control and (e)-(h) DM1 immortalized transdifferentiated myoblast-like cells encapsulated in GelMA-CMCMA hydrogels. (a), (e) Nuclei were stained with DAPI (blue). (b), (f) Nuclear RNA aggregates of expanded-CUG repeats (white arrows) were only present in DM1 cells and detected by FISH using a Cy3-(CAG) 7 -Cy3 probe (red). (c), (g) MBNL1 stainings (green) showing a reduced expression of MBNL1 in DM1 cells compared to controls. (d), (h) Merged images showing colocalization of MBNL1 with nuclear CUGexp-RNA aggregates in DM1 transdifferentiated myoblast-like cells (Scale bars: $10 \mu \mathrm{m}$ ).

Then, the 3D reconstructions of myotubes expressing SAA (figures 6(a)-(f)) were used to measure the individual diameter of control and DM1 myotubes over time. Several works have quantified myotube diameter in 2D for different purposes; for example, to characterize patient cells [75], test different culture conditions [76], evaluate the influence of topography in differentiation [77], or to study the effect of administrating a given compound to myotubes [78]. Myotube diameter has also been measured in 3D cultures; for instance, Khodabukus et al compared myotube cross-sections to analyze the maturation of electrically stimulated constructs [79]. Measurements in all these cases were carried out manually, using tools from ImageJ software. In contrast, our measurements were obtained by a $3 \mathrm{D}$ reconstruction using Imaris software (figure 6(g)). With this reconstruction, each myotube diameter was automatically obtained with surface analysis tools. We found that 3D DM1 myotubes have a reduced diameter compared to healthy control myotubes (13.4 vs. $21.7 \mu \mathrm{m}$ ) after the first week of 3D culture (figure 6(h)). After two weeks of culture, both types of myotubes increased in size, but DM1 myotubes continue to show a reduced diameter compared to control myotubes. On the contrary, after three weeks of culture, we did not detect differences between the size of $3 \mathrm{D}$ myotubes. Nevertheless, this observation might not be due to a DM1 phenotype, but the healthy myotubes have a reduced size after three weeks as a potential senescence of the cultures. For this reason, assays with these $3 \mathrm{D}$ muscle models should be performed in myotubes cultured for less than three weeks.
Previous works in DM1 cell models have described deficient muscle differentiation. A reduced fusion potential has been observed in $2 \mathrm{D}$ studies with myoblasts from transgenic mice [80] and myogenic satellite cells from DM1 patients [81]. As an indicator of muscle differentiation, the fusion index has been used to investigate some aspects of DM1 molecular pathology $[82,83]$. It has also been applied to compare DM1 vs. control cells while establishing cell lines [48] and test if proposed treatments could restore muscle differentiation [84]. Notably, we demonstrated that $3 \mathrm{D}$ micropatterning improves the differentiation of DM1 cells, obtaining fusion index values that are similar to healthy controls. Thus, this indicator cannot be applied to evaluate phenotype rescue by a given therapy. Instead, we found a significant difference in the diameter of 3D myotubes from DM1 and control cells. DM1 usually presents in adulthood, when tissue is already formed. Therefore, from a drug development perspective, the reduced myotube diameter in $3 \mathrm{D}$ is a more physiologically relevant phenotype than impaired fusion in $2 \mathrm{D}$ cultures. Considering these results, we propose that this new structural phenotype would be a better indicator to assess the effects of DM1 therapies in preclinical research.

\subsection{AntagomiR treatments rescue bioengineered 3D DM1 muscle phenotypes}

Antisense oligonucleotides for specific blocking of miR-23b (antagomiRs) have already shown efficacy to increase MBNL protein levels in 2D DM1 myoblasts [46] as well as to rescue molecular pathology, splicing events, and functional phenotypes in a mouse 

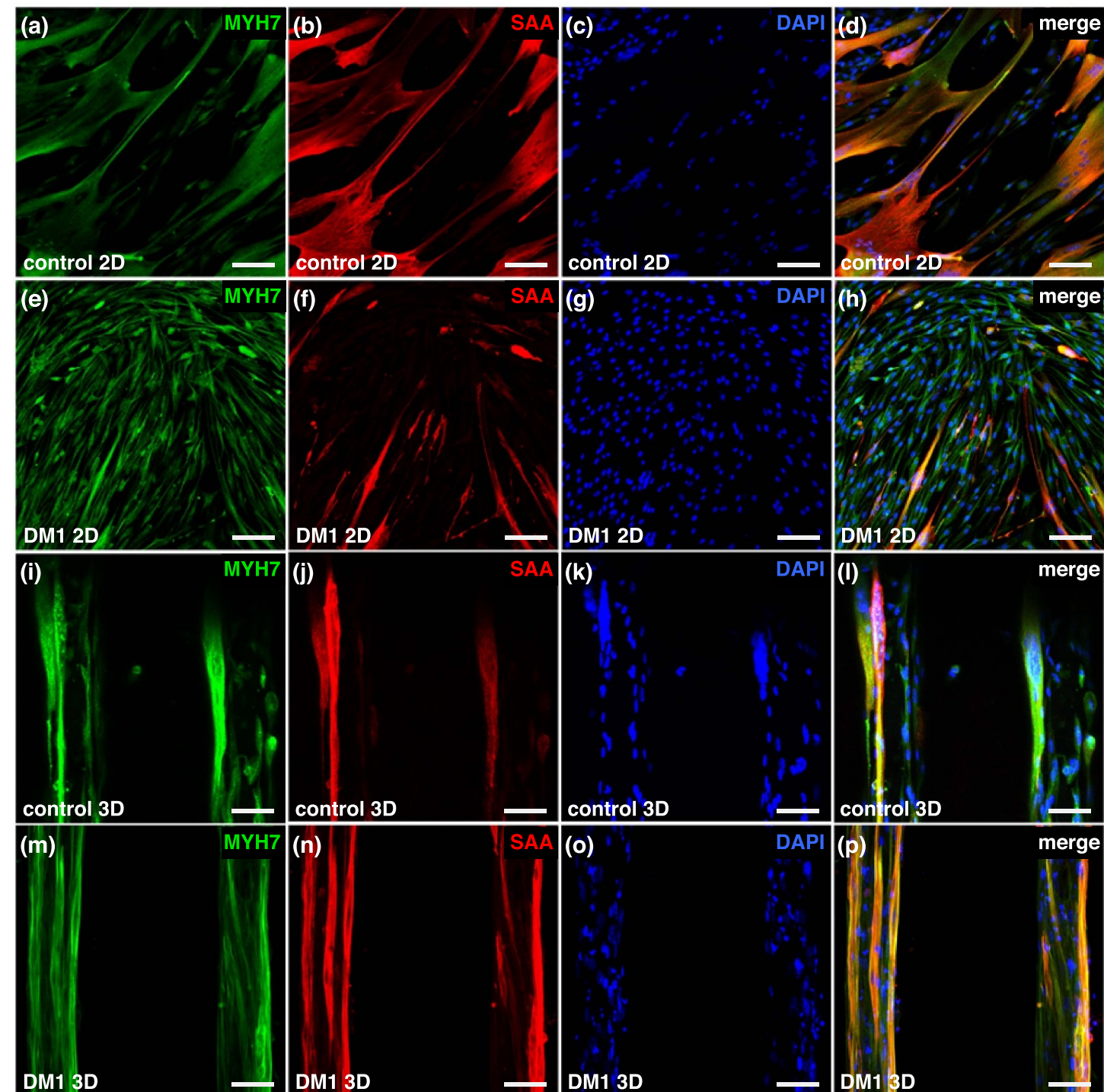

(q)
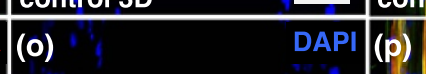

merge
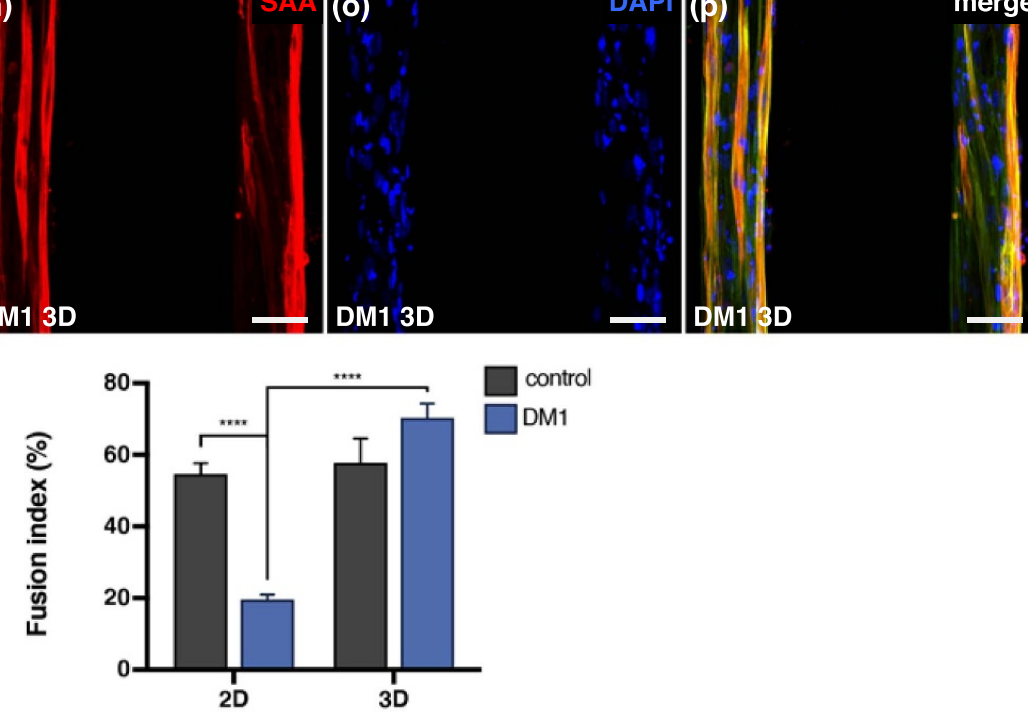

Figure 5. Cell differentiation is improved in 3D cultures. (a)-(p) Representative confocal images of 2D (a)-(h) and 3D (i) $-(\mathrm{p})$ cultures stained for myosin heavy chain 7 (MYH7, green), sarcomeric $\alpha$-actinin (SAA, red), and nuclei (DAPI, blue) after seven days in differentiation conditions (scale bars: $50 \mu \mathrm{m}$ ). (q) Graph showing comparison of fusion index expressed as the percentage of differentiated myotubes with respect to the total cell number. ${ }^{* * * *} \mathrm{p}<0.0001$.

model $[46,47]$.To demonstrate the application of our bioengineered in vitro model of DM1 in preclinical research, we evaluated the effect of this antagomiR in our 3D cell cultures.

AntagomiR against miR-23b ( $\alpha$-miR-23b) or a scrambled control (sc-control) antagomiR were administered to bioengineered DM1 microtissues by gymnotic delivery at a concentration of $100 \mathrm{nM}$. Of note, cells were already orientated along the micropatterns and transdifferentiated into myoblast-like cells at the moment of antagomiRs administration. The treatment lasted for seven days. To determine the success of the treatment, we analyzed the expression levels of miR-23b by real-time PCR (figure $7(a)$ ). We observed a reduction of miR23b expression in DM1 3D muscles treated with $\alpha$ miR-23b compared to the DM1 3D muscles treated with sc-control antagomiR. Next, we investigated if silencing miR-23b enhanced MBNL transcripts in the bioengineered $3 \mathrm{D}$ microtissues. We found an 


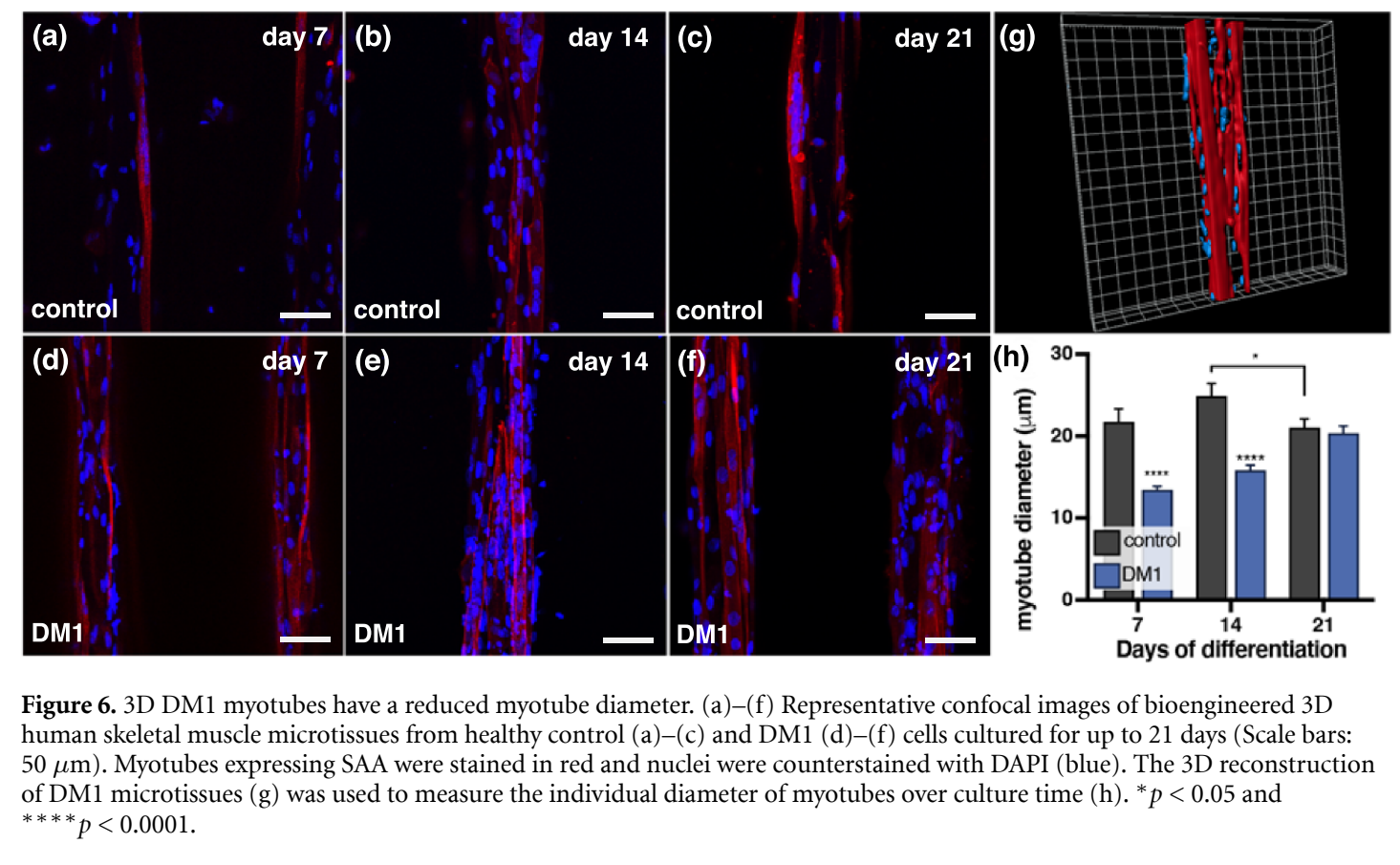

increase in MBNL2 transcripts in 3D DM1 myotubes treated with $\alpha$-miR-23b compared to sc-controltreated tissues. However, MBNL1 was not increased at the transcriptional level (figure $7(\mathrm{~b})$ ). Of note, western blot quantification showed an increase in both MBNL1-2 proteins in bioengineered microtissues treated with $\alpha$-miR-23b (figures 7 (c) and (d)). To determine if the increase of MBNL proteins rescue characteristic DM1 spliceopathy, we analyzed the exon inclusion (Percentage Spliced In; PSI) of two MBNL1-dependent splicing events: BIN1 and NFIX; and one MBNL2-dependent splicing event: SPTAN1. Remarkably, the exon inclusion was significantly rescued for the three splicing events (figures 7(e) and (f)). These rescue levels are lower than those obtained by transfection of antagomiRs in 2D myoblasts in previous studies [46]. This can be explained by the different delivery strategies used. Gene silencing by oligonucleotides usually requires a delivery vehicle to perform cell transfection. This requirement comes with several challenges that can complicate the development of oligonucleotide therapies [85]. As an alternative, gymnotic delivery ('naked' delivery of oligonucleotides to cells) has been promising in recent years. With this delivery approach, cells in culture are exposed to a constant concentration of oligonucleotides, similar to what happens in vivo when plasma and tissues are exposed to saline-formulated oligonucleotides. Transfection strategy allows the internalization of large amounts of oligonucleotides. Nevertheless, gymnotic delivery remains a more physiologically relevant delivery approach, and its effects can be improved by dosage optimization in future assays.
Finally, to assess the effect of antagomiRs on the DM1 3D structural phenotype, we measured the diameter of 3D myotubes treated with $\alpha$-miR-23b and compared them to sc-control-treated myotubes (figure $7(\mathrm{~g})$ ). After seven days of treatment, we found that sc-control-treated microtissues had a mean myotube diameter of $14.4 \mu \mathrm{m}$, similar to DM1 myotubes without any treatment. Remarkably, upon treatment with $\alpha$-miR-23b, myotubes reached a mean diameter of $21.9 \mu \mathrm{m}$ (figure $7(\mathrm{~h})$ ), comparable to the diameter of myotubes from healthy control cells (figure 6(h)). Altogether, these results highlight the application of our bioengineered in vitro 3D model to assess DM1 therapies. Other antisense oligonucleotides and other potential therapies like small molecules or peptides can be tested on this platform. Furthermore, drug effects can be analyzed at different levels, including transcriptional, post-transcriptional, and structural phenotype changes.

\section{Conclusions}

In this work, we described a detailed protocol for fabricating 3D skeletal muscle microtissues that can be maintained in long-lasting cultures. This micromolding technique is cheap and can be easily reproduced. Importantly, cell encapsulation in GelMACMCMA micropatterned hydrogels produce highly aligned multinucleated myotubes. Using this protocol, we generated for the first time a bioengineered human 3D skeletal muscle model for myotonic dystrophy. Remarkably, this protocol can be applied to cells from patients for different muscle diseases 
(a)

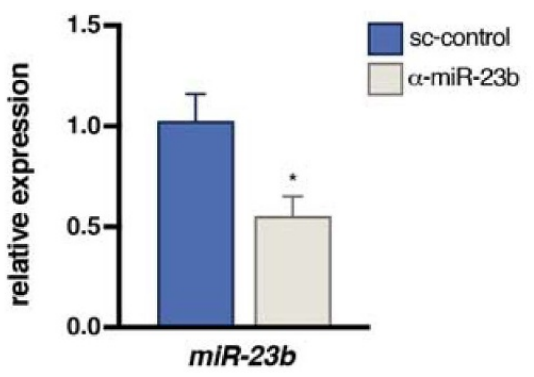

(c)
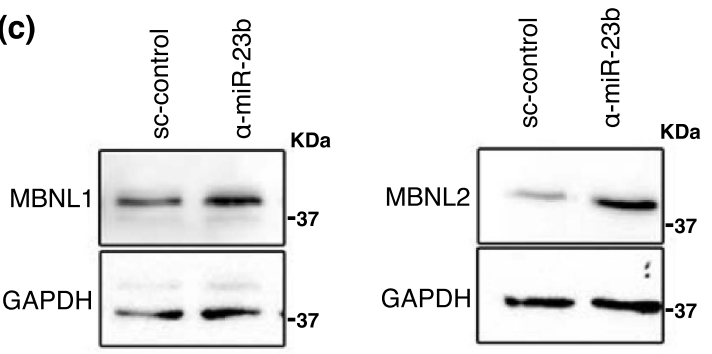

(e)
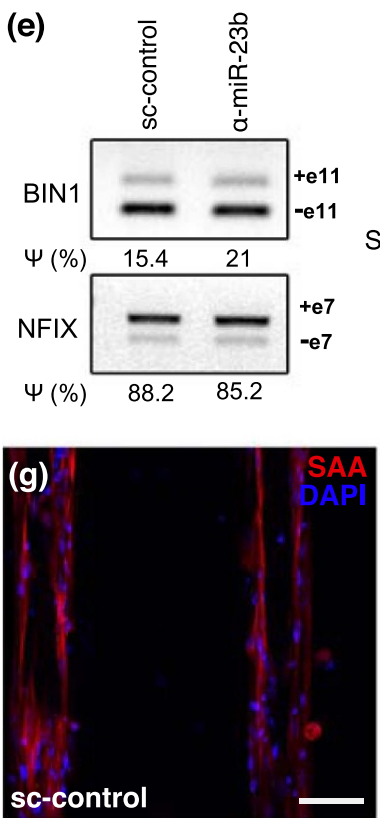

(b)

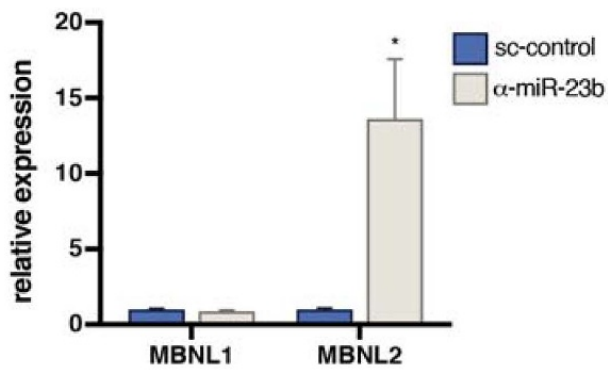

(d)

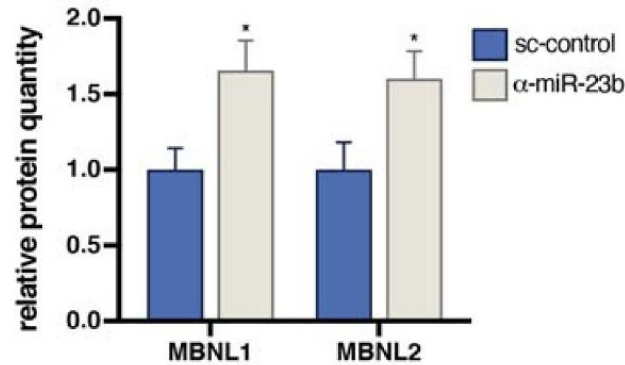

(f)
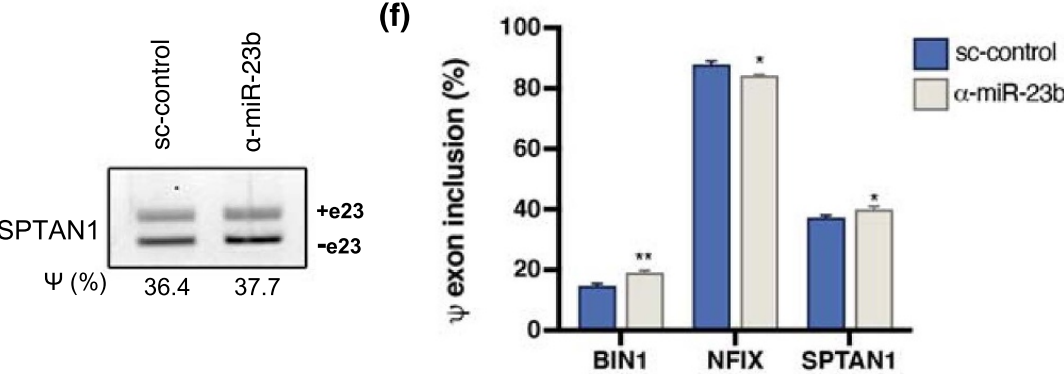

(i)

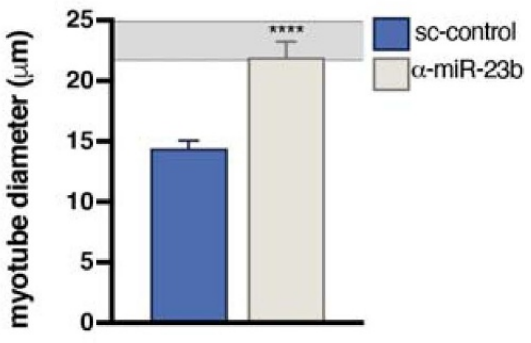

Figure 7. Therapeutic effect of antagomiR-23b on a bioengineered in vitro 3D model of DM1 human skeletal muscle. (a) Real-time PCR quantification of miR-23b expression after seven days of treatment with $100 \mathrm{nM}$ of antagomiR-23b $(\alpha$-miR-23b) or scrambled control antagomiR (sc-control). $U 1$ and $U 6$ snRNAs were used as reference genes. (b) qRT-PCR quantification of MBNL1 and MBNL2 expression relative to GAPDH and ACTB genes. (c), (d) Western blot quantification of MBNL1 and MBNL2 protein expression levels in 3D DM1 myotubes seven days after treatment with $100 \mathrm{nM}$ of $\alpha$-miR-23b or sc-control. GAPDH expression was used as endogenous control. (e), (f) RT-PCR analyses of splicing events altered in BIN1, NFIX, and SPTAN1 in 3D DM1 myotubes. (g), (h) Confocal images of DM1 myotubes after treatment with sc-control (g) or $\alpha$-miR-23b (h). Myotubes expressing SAA were stained in red and nuclei were counterstained with DAPI (blue) (scale bars: $50 \mu \mathrm{m}$ ). (i) Quantification of the diameter of 3D DM1 myotubes treated with sc-control or $\alpha$-miR-23b. The shaded area corresponds to the mean of myotube diameter of healthy control myotubes after 7 (bottom limit) and 14 days (top limit) of differentiation. ${ }^{*} p<0.05,{ }^{* *} p<0.01$ and $* * * * p<0.0001$.

(i.e. other muscular dystrophies) to obtain patientspecific models. DM1 patient-derived cells encapsulated in GelMA-CMCMA hydrogels present DM1 molecular characteristics and correctly differentiated into multinucleated myotubes. A reduced fusion index, an indicator of muscle differentiation, is a typical feature of DM1 standard 2D cultures. Notably, the fusion index is restored to control values in DM1 3D myotubes. However, DM1 3D microtissues display a new structural phenotype; the 3D diameter of myotubes is significantly thinner than control myotubes. Therefore, this more physiological phenotype of the bioengineered 3D DM1 myotubes can be used for drug screening studies of DM1. To evaluate the applicability of this in vitro model in evaluating therapeutic compounds, we treated the bioengineered 3D microtissues with antisense oligonucleotides against miR-23b. Our findings 
indicated that antagomiRs for miR-23b are able to rescue molecular and structural phenotypes of DM1 disease when administrated to bioengineered $3 \mathrm{D}$ muscle. Overall, we demonstrated that our patientderived 3D model is a relevant alternative to the existing in vitro and animal models. These bioengineered 3D skeletal muscle microtissues could be useful in a preclinical platform for DM1 drug development.

\section{Data availability statement}

All data that support the findings of this study are included within the article (and any supplementary files).

\section{Acknowledgments}

The authors thank the technical support of Judith Linacero, from the Microscopy and MicroFabSpace Characterization Facilities (IBEC), for her advice and for performing SEM imaging. This project received financial support from the European Research Council program under Grants ERC-StGDAMOC (714317), the Spanish Ministry of Economy and Competitiveness, through the 'Severo Ochoa' Program for Centres of Excellence in R\&D (SEV2016-2019) and 'Retos de investigación: Proyectos $\mathrm{I}+\mathrm{D}+\mathrm{i}$ ' (TEC2017-83716-C2-1-R and TEC201783716-C2-2-R), the CERCA Programme/Generalitat de Catalunya (2014-SGR-1460) and Fundación Bancaria 'la Caixa'-Obra Social 'la Caixa' (project IBEC-La Caixa Healthy Ageing) to J R-A.

X F-G was supported by a predoctoral fellowship (BES-2016-076681) from the Ministerio de Economia y Competitividad. J M F-C was awarded with postdoctoral fellowship (APOSTD/2017/088) from the Generalitat Valenciana. E C-H was awarded with postdoctoral fellowship (APOSTD/2019/142) from the Generalitat Valenciana. Additional support was received from the Generalitat Valenciana (PROMETEO/2020/081) to R A. Cells used in this study were kindly provided by Dr Denis Furling and Dr Vincent Mouly (Institute of Myology, Paris). Antibody MB1a (4A8) and antibody MB2a (3B4) was provided by MDA Monoclonal Antibody Resources.

\section{Author contributions}

X F-G, J M F-C, R A, and J R-A conceived this work. $X$ F-G and J M F-C performed and coordinated the experiments and data analysis. M A O designed and fabricated the micropatterned silicon molds, E C-H did the molecular validation of antagomiR treatments, J C and E M performed the AFM measurements and data analysis. J R-A, R A and E M acquired the funding for this project. X F-G and J M F-C prepared the manuscript with the supervision of $\mathrm{J}$ R-A and input from all the authors.

\section{Ethical statement}

The authors declared no potential conflicts of interest with respect to the research, authorship, and/or publication of this article.

\section{ORCID iDs}

Xiomara Fernández-Garibay (1)

https://orcid.org/0000-0002-0697-985X

Elena Martínez (1) https://orcid.org/0000-0002-

6585-4213

Juan M Fernández-Costa (ํ) https://orcid.org/00000002-1854-6082

Javier Ramón-Azcón (ib https://orcid.org/0000-00023636-8013

\section{References}

[1] Csapo R, Gumpenberger M and Wessner B 2020 Skeletal muscle extracellular matrix-what do we know about its composition, regulation, and physiological roles? A narrative review Front. Physiol. 11 1-15

[2] Meriggioli M N and Sanders D B 2009 Autoimmune myasthenia gravis: emerging clinical and biological heterogeneity Lancet Neurol. 8 475-90

[3] Kaczor J J, Robertshaw H A and Tarnopolsky M A 2017 Higher oxidative stress in skeletal muscle of McArdle disease patients Mol. Genet. Metab. Rep. 12 69-75

[4] DeFronzo R A and Tripathy D 2009 Skeletal muscle insulin resistance is the primary defect in type 2 diabetes Diabetes Care 32 S157-63

[5] Corcoran M P, Lamon-Fava S and Fielding R A 2007 Skeletal muscle lipid deposition and insulin resistance: effect of dietary fatty acids and exercise Am. J. Clin. Nutr. 85 662-77

[6] Mercuri E, Bönnemann C G and Muntoni F 2019 Muscular dystrophies Lancet 394 2025-38

[7] Benarroch L, Bonne G, Rivier F and Hamroun D 2019 The 2020 version of the gene table of neuromuscular disorders (nuclear genome) Neuromuscul. Disord. 29 980-1018

[8] Carter J C, Sheehan D W, Prochoroff A and Birnkrant D J 2018 Muscular dystrophies Clin. Chest Med. 39 377-89

[9] Bersini S, Arrigoni C, Lopa S, Bongio M, Martin I and Moretti M 2016 Engineered miniaturized models of musculoskeletal diseases Drug Discov. Today 21 1429-36

[10] Khodabukus A and Baar K 2016 Factors that affect tissue-engineered skeletal muscle function and physiology Cells Tissues Organs 202 159-68

[11] Taylor M J, Tanna S and Sahota T 2010 In vivo study of a polymeric glucose-sensitive insulin delivery system using a rat model J. Pharm. Sci. 99 4215-27

[12] DiMasi J A, Grabowski H G and Hansen R W 2016 Innovation in the pharmaceutical industry: new estimates of R\&D costs J. Health Econ. 47 20-33

[13] Dowden H and Munro J 2019 Trends in clinical success rates and therapeutic focus Nat. Rev. Drug Discov. 18 495-6

[14] Moyle L A, Jacques E and Gilbert P M 2020 Engineering the next generation of human skeletal muscle models: from cellular complexity to disease modeling Curr. Opin. Biomed. Eng. 16 9-18

[15] Chal J and Pourquié O 2017 Making muscle: skeletal myogenesis in vivo and in vitro Development 144 2104-22

[16] Smoak M M and Mikos A G 2020 Advances in biomaterials for skeletal muscle engineering and obstacles still to overcome Mater. Today Bio 7100069

[17] Zhang Y S and Khademhosseini A 2017 Advances in engineering hydrogels Science 356 eaaf3627 
[18] Ahadian S et al 2015 Hybrid hydrogels containing vertically aligned carbon nanotubes with anisotropic electrical conductivity for muscle myofiber fabrication Sci. Rep. 44271

[19] García-Lizarribar A, Fernández-Garibay X Velasco-Mallorquí F, Castaño A G, Samitier J and Ramon-Azcon J 2018 Composite biomaterials as long-lasting scaffolds for 3D bioprinting of highly aligned muscle tissue Macromol. Biosci. 181800167

[20] Ortega M A, Fernández-Garibay X, Castaño A G, De Chiara F, Hernández-Albors A, Balaguer-Trias J and Ramón-Azcón J 2019 Muscle-on-a-chip with an on-site multiplexed biosensing system for in situ monitoring of secreted IL-6 and TNF- $\alpha$ Lab Chip 19 2568-80

[21] Hutson C B, Nichol J W, Aubin H, Bae H, Yamanlar S, Al-Haque S, Koshy S T and Khademhosseini A 2011 Synthesis and characterization of tunable poly(ethylene glycol): gelatin methacrylate composite hydrogels Tissue Eng. A 17 1713-23

[22] Ozimki L, Sabater-Arcis M, Bargiela A and Artero R 2020 The hallmarks of myotonic dystrophy type 1 muscle dysfunction Biol. Rev. 96 716-30

[23] Udd B and Krahe R 2012 The myotonic dystrophies: molecular, clinical, and therapeutic challenges Lancet Neurol. 11 891-905

[24] Wang E T et al 2019 Transcriptome alterations in myotonic dystrophy skeletal muscle and heart Hum. Mol. Genet. 28 1312-21

[25] Ebralidze A 2004 RNA leaching of transcription factors disrupts transcription in myotonic dystrophy Science 303 383-7

[26] Jones K, Wei C, Iakova P, Bugiardini E, Schneider-Gold C, Meola G, Woodgett J, Killian J, Timchenko N A and Timchenko L T 2012 GSK $3 \beta$ mediates muscle pathology in myotonic dystrophy J. Clin. Invest. 122 4461-72

[27] Rau F et al 2011 Misregulation of miR-1 processing is associated with heart defects in myotonic dystrophy Nat. Struct. Mol. Biol. 18 840-5

[28] Krol J, Fiszer A, Mykowska A, Sobczak K, De Mezer M and Krzyzosiak W J 2007 Ribonuclease dicer cleaves triplet repeat hairpins into shorter repeats that silence specific targets Mol. Cell 25 575-86

[29] Fernandez-Costa J M et al 2013 Expanded CTG repeats trigger miRNA alterations in Drosophila that are conserved in myotonic dystrophy type 1 patients Hum. Mol. Genet. 22 704-16

[30] Kalsotra A, Singh R K, Gurha P, Ward A J, Creighton C J and Cooper T A 2014 The Mef2 transcription network is disrupted in myotonic dystrophy heart tissue, dramatically altering miRNA and mRNA expression Cell Rep. 6 336-45

[31] Du H et al 2010 Aberrant alternative splicing and extracellular matrix gene expression in mouse models of myotonic dystrophy Nat. Struct. Mol. Biol. 17 187-93

[32] Charizanis K et al 2012 Muscleblind-like 2-mediated alternative splicing in the developing brain and dysregulation in myotonic dystrophy Neuron 75 437-50

[33] Goodwin M et al 2015 MBNL sequestration by toxic RNAs and RNA misprocessing in the myotonic dystrophy brain Cell Rep. 12 1159-68

[34] Batra R et al 2014 Loss of MBNL leads to disruption of developmentally regulated alternative polyadenylation in RNA-mediated disease Mol. Cell 56 311-22

[35] Fernandez-Costa J M, Llamusi M B, Garcia-Lopez A and Artero R 2011 Alternative splicing regulation by Muscleblind proteins: from development to disease Biol. Rev. 86 947-58

[36] Gomes-Pereira M, Cooper T A and Gourdon G 2011 Myotonic dystrophy mouse models: towards rational therapy development Trends Mol. Med. 17 506-17

[37] Cerro-Herreros E, Fernandez-Costa J M, Sabater-Arcis M, Llamusi B and Artero R 2016 Derepressing muscleblind expression by miRNA sponges ameliorates myotonic dystrophy-like phenotypes in Drosophila Sci. Rep. 6 1-13

[38] Bargiela A, Cerro-Herreros E, Fernandez-Costa J M, Vilchez J J, Llamusi B and Artero R 2015 Increased autophagy and apoptosis contribute to muscle atrophy in a myotonic dystrophy type 1 Drosophila model DMM Dis. Model. Mech. 8 679-90

[39] Garcia-Lopez A, Monferrer L, Garcia-Alcover I, Vicente-Crespo M, Alvarez-Abril M C and Artero R D 2008 Genetic and chemical modifiers of a CUG toxicity model in drosophila PLoS One 3 e1595

[40] Todd P K, Ackall F Y, Hur J, Sharma K, Paulson H L and Dowling J J 2014 Transcriptional changes and developmental abnormalities in a zebrafish model of myotonic dystrophy type 1 DMM Dis. Model. Mech. 7 143-55

[41] Chen G, Masuda A, Konishi H, Ohkawara B, Ito M, Kinoshita M, Kiyama H, Matsuura T and Ohno K 2016 Phenylbutazone induces expression of MBNL1 and suppresses formation of MBNL1-CUG RNA foci in a mouse model of myotonic dystrophy Sci. Rep. 6 1-11

[42] Konieczny P, Selma-Soriano E, Rapisarda A S, Fernandez-Costa J M, Perez-Alonso M and Artero R 2017 Myotonic dystrophy: candidate small molecule therapeutics Drug Discov. Today 22 1740-8

[43] Huang K, Masuda A, Chen G, Bushra S, Kamon M, Araki T, Kinoshita M, Ohkawara B, Ito M and Ohno K 2020 Inhibition of cyclooxygenase-1 by nonsteroidal anti-inflammatory drugs demethylates MeR2 enhancer and promotes Mbnll transcription in myogenic cells Sci. Rep. 102558

[44] Overby S J, Cerro-Herreros E, Llamusi B and Artero R 2018 RNA-mediated therapies in myotonic dystrophy Drug Discov. Today 23 2013-22

[45] López Castel A, Overby S J and Artero R 2019 MicroRNA-based therapeutic perspectives in myotonic dystrophy Int. J. Mol. Sci. 205600

[46] Cerro-Herreros E, Sabater-Arcis M, Fernandez-Costa J M, Moreno N, Perez-Alonso M, Llamusi B and Artero R 2018 miR-23b and miR-218 silencing increase Muscleblind-like expression and alleviate myotonic dystrophy phenotypes in mammalian models Nat. Commun. 92482

[47] Cerro-Herreros E, González-Martínez I, Moreno-Cervera N, Overby S, Pérez-Alonso M, Llamusí B and Artero R 2020 Therapeutic potential of AntagomiR-23b for treating myotonic dystrophy Mol. Ther. Nucleic Acids 21 837-49

[48] Arandel L et al 2017 Immortalized human myotonic dystrophy muscle cell lines to assess therapeutic compounds Dis. Model. Mech. 10 487-97

[49] Alcaraz J, Buscemi L, Grabulosa M, Trepat X, Fabry B, Farré R and Navajas D 2003 Microrheology of human lung epithelial cells measured by atomic force microscopy Biophys. J. 84 2071-9

[50] Comelles J, Fernández-Majada V, Berlanga-Navarro N, Acevedo V, Paszkowska K and Martínez E 2020 Microfabrication of poly(acrylamide) hydrogels with independently controlled topography and stiffness Biofabrication 12025023

[51] Schindelin J et al 2012 Fiji: an open-source platform for biological-image analysis Nat. Methods 9 676-82

[52] Schneider C A, Rasband W S and Eliceiri K W 2012 NIH image to ImageJ: 25 years of image analysis Nat. Methods 9 671-5

[53] Peltier H J and Latham G J 2008 Normalization of microRNA expression levels in quantitative RT-PCR assays: identification of suitable reference RNA targets in normal and cancerous human solid tissues RNA 14 844-52

[54] Fernández-Costa J M, Fernández-Garibay X, Velasco-Mallorquí F and Ramón-Azcón J 2021 Bioengineered in vitro skeletal muscles as new tools for muscular dystrophies preclinical studies J. Tissue Eng. $121-19$

[55] Aubin H, Nichol J W, Hutson C B, Bae H, Sieminski A L, Cropek D M, Akhyari P and Khademhosseini A 2010 Directed 3D cell alignment and elongation in microengineered hydrogels Biomaterials 31 6941-51 
[56] Costantini M, Testa S, Fornetti E, Barbetta A, Trombetta M, Cannata S M, Gargioli C and Rainer A 2017 Engineering muscle networks in 3D gelatin methacryloyl hydrogels: influence of mechanical stiffness and geometrical confinement Front. Bioeng. Biotechnol. 5 1-8

[57] Kang H W, Lee S J, Ko I K, Kengla C, Yoo J J and Atala A 2016 A 3D bioprinting system to produce human-scale tissue constructs with structural integrity Nat. Biotechnol. 34 312-9

[58] Rouwkema J, Koopman B F J M, C A Van B, Dhert W J A and Malda J 2009 Supply of nutrients to cells in engineered tissues Biotechnol. Genet. Eng. Rev. 26 163-78

[59] Peter M and Tayalia P 2016 An alternative technique for patterning cells on poly(ethylene glycol) diacrylate hydrogels RSC Adv. 6 40878-85

[60] Nichol J W, Koshy S T, Bae H, Hwang C M, Yamanlar S and Khademhosseini A 2010 Cell-laden microengineered gelatin methacrylate hydrogels Biomaterials 31 5536-44

[61] Wong D Y, Ranganath T and Kasko A M 2015 Low-dose, long-wave UV light does not affect gene expression of human mesenchymal stem cells PLoS One 10 1-21

[62] Fairbanks B D, Schwartz M P, Bowman C N and Anseth K S 2009 Photoinitiated polymerization of PEG-diacrylate with lithium phenyl-2,4,6-trimethylbenzoylphosphinate: polymerization rate and cytocompatibility Biomaterials $306702-7$

[63] Ouyang L, Highley C B, Sun W and Burdick J A 2017 A generalizable strategy for the 3D bioprinting of hydrogels from nonviscous photo-crosslinkable inks Adv. Mater. 291604983

[64] Boere K W M, Visser J, Seyednejad H, Rahimian S, Gawlitta D, Van Steenbergen M J, Dhert W J A, Hennink W E, Vermonden T and Malda J 2014 Covalent attachment of a three-dimensionally printed thermoplast to a gelatin hydrogel for mechanically enhanced cartilage constructs Acta Biomater. 10 2602-11

[65] Wang J, Khodabukus A, Rao L, Vandusen K, Abutaleb N and Bursac N 2019 Engineered skeletal muscles for disease modeling and drug discovery Biomaterials 221119416

[66] Bigot A, Klein A F, Gasnier E, Jacquemin V, Ravassard P, Butler-Browne G, Mouly V and Furling D 2009 Large CTG repeats trigger p16-dependent premature senescence in myotonic dystrophy type 1 muscle precursor cells Am. J. Pathol. 174 1435-42

[67] Reeves R, Ribeiro A, Lombardo L, Boyer R and Leach J B 2010 Synthesis and characterization of carboxymethylcellulose-methacrylate hydrogel cell scaffolds Polymers 2 252-64

[68] Hernández-Albors A, Castaño A G, Fernández-Garibay X, Ortega M A, Balaguer J and Ramón-Azcón J 2019 Microphysiological sensing platform for an in-situ detection of tissue-secreted cytokines Biosens. Bioelectron. X 2100025

[69] Engler A J, Griffin M A, Sen S, Bönnemann C G, Sweeney H L and Discher D E 2004 Myotubes differentiate optimally on substrates with tissue-like stiffness J. Cell Biol. $166877-87$

[70] Gilbert P M, Havenstrite K L, Magnusson K E G, Sacco A, Leonardi N A, Kraft P, Nguyen N K, Thrun S, Lutolf M P and Blau H M 2010 Substrate elasticity regulates skeletal muscle stem cell self-renewal in culture Science 329 1078-81

[71] Chiron S, Tomczak C, Duperray A, Lainé J, Bonne G, Eder A, Hansen A, Eschenhagen T, Verdier C and Coirault C 2012
Complex interactions between human myoblasts and the surrounding 3D fibrin-based matrix PLoS One 7 2-9

[72] Van Vlierberghe S, Cnudde V, Dubruel P, Masschaele B, Cosijns A, De Paepe I, Jacobs P J S, Van Hoorebeke L, Remon J P and Schacht E 2007 Porous gelatin hydrogels: 1. cryogenic formation and structure analysis Biomacromolecules 8 331-7

[73] Miri A K, Hosseinabadi H G, Cecen B, Hassan S and Zhang Y S 2018 Permeability mapping of gelatin methacryloyl hydrogels Acta Biomater. 77 38-47

[74] Monge C, Ren K, Berton K, Guillot R, Peyrade D and Picart C 2012 Engineering muscle tissues on microstructured polyelectrolyte multilayer films Tissue Eng. A 18 1664-76

[75] Pomiès $\mathrm{P}$, Rodriguez J, Blaquière $\mathrm{M}$, Sedraoui S, Gouzi F, Carnac G, Laoudj-Chenivesse D, Mercier J, Préfaut C and Hayot M 2015 Reduced myotube diameter, atrophic signalling and elevated oxidative stress in cultured satellite cells from COPD patients J. Cell. Mol. Med. 19 175-86

[76] Archer-Lahlou E, Lan C and Jagoe R T 2018 Physiological culture conditions alter myotube morphology and responses to atrophy treatments: implications for in vitro research on muscle wasting Physiol. Rep. 6 e13726

[77] Almonacid Suarez A M, Zhou Q, Rijn P and Harmsen M C 2019 Directional topography gradients drive optimum alignment and differentiation of human myoblasts J. Tissue Eng. Regen. Med. 13 2234-45

[78] Ohno Y, Oyama A, Kaneko H, Egawa T, Yokoyama S, Sugiura T, Ohira Y, Yoshioka T and Goto K 2018 Lactate increases myotube diameter via activation of MEK/ERK pathway in C2C12 cells Acta Physiol. 223 e13042

[79] Khodabukus A, Madden L, Prabhu N K, Koves T R, Jackman C P, Muoio D M and Bursac N 2019 Electrical stimulation increases hypertrophy and metabolic flux in tissue-engineered human skeletal muscle Biomaterials 198 259-69

[80] Timchenko N A, Patel R, Iakova P, Cai Z J, Quan L and Timchenko L T 2004 Overexpression of CUG triplet repeat-binding protein, CUGBP1, in mice inhibits myogenesis J. Biol. Chem. 279 13129-39

[81] Furling D, Lemieux D, Taneja K and Puymirat J 2001 Decreased levels of myotonic dystrophy protein kinase (DMPK) and delayed differentiation in human myotonic dystrophy myoblasts Neuromuscul. Disord. 11 728-35

[82] Peng X, Shen X, Chen X, Liang R, Azares A R and Liu Y 2015 Celf1 regulates cell cycle and is partially responsible for defective myoblast differentiation in myotonic dystrophy RNA toxicity Biochim. Biophys. Acta 1852 1490-7

[83] Beaulieu D, Thebault P, Pelletier R, Chapdelaine P, Tarnopolsky M, Furling D and Puymirat J 2012 Abnormal prostaglandin E2 production blocks myogenic differentiation in myotonic dystrophy Neurobiol. Dis. 45 122-9

[84] François V, Klein A F, Beley C, Jollet A, Lemercier C, Garcia L and Furling D 2011 Selective silencing of mutated mRNAs in DM1 by using modified hU7-snRNAs Nat. Struct. Mol. Biol. 18 85-7

[85] Soifer H S, Koch T, Lai J, Hansen B, Hoeg A, Oerum H and Stein C A 2012 Silencing of gene expression by gymnotic delivery of antisense oligonucleotides Functional Genomics: Methods in Molecular Biology (Methods and Protocols vol 815) ed M Kaufmann and C Klinger (New York: Springer) pp 333-46 